\title{
Chronic Treatment with the AMPK Agonist AICAR Prevents Skeletal Muscle Pathology but Fails to Improve Clinical Outcome in a Mouse Model of Severe Spinal Muscular Atrophy
}

\author{
Clàudia Cerveró $^{1} \cdot$ Neus Montull $^{1} \cdot$ Olga Tarabal $^{1} \cdot$ Lídia Piedrafita $^{1}$. \\ Josep E. Esquerda ${ }^{1} \cdot$ Jordi Calderó $^{1}$
}

Published online: 18 November 2015

(C) The American Society for Experimental NeuroTherapeutics, Inc. 2015

\begin{abstract}
Spinal muscular atrophy (SMA) is a genetic neuromuscular disorder characterized by spinal and brainstem motor neuron (MN) loss and skeletal muscle paralysis. Currently, there is no effective treatment other than supportive care to ameliorate the quality of life of patients with SMA. Some studies have reported that physical exercise, by improving muscle strength and motor function, is potentially beneficial in SMA. The adenosine monophosphate-activated protein kinase agonist 5aminoimidazole-4-carboxamide-1- $\beta$-D-ribofuranoside (AICAR) has been reported to be an exercise mimetic agent that is able to regulate muscle metabolism and increase endurance both at rest and during exercise. Chronic AICAR administration has been shown to ameliorate the dystrophic muscle phenotype and motor behavior in the $m d x$ mouse, a model of Duchenne muscular dystrophy. Here, we investigated whether chronic AICAR treatment was able to elicit beneficial effects on motor abilities and neuromuscular histopathology in a mouse model of severe SMA (the SMN $\Delta 7$ mouse). We report that AICAR improved skeletal muscle atrophy and structural changes found in neuromuscular junctions of $\operatorname{SMN} \Delta 7$ animals. However, although AICAR prevented the loss of glutamatergic excitatory synapses on MNs, this compound
\end{abstract}

Jordi Calderó

jordi.caldero@mex.udl.cat

1 Unitat de Neurobiologia Cel·lular, Departament de Medicina Experimental, Facultat de Medicina, Universitat de Lleida and Institut de Recerca Biomèdica de Lleida (IRBLLEIDA), Av. Rovira Roure 80, 25198 Lleida, Catalonia, Spain was not able to mitigate $\mathrm{MN}$ loss or the microglial and astroglial reaction occurring in the spinal cord of diseased mice. Moreover, no improvement in survival or motor performance was seen in $\mathrm{SMN} \Delta 7$ animals treated with AICAR. The beneficial effects of AICAR in SMA found in our study are SMN-independent, as no changes in the expression of this protein were seen in the spinal cord and skeletal muscle of diseased animals treated with this compound.

Keywords AICAR $\cdot$ Spinal muscular atrophy $\cdot \operatorname{SMN} \Delta 7$ mouse $\cdot$ Skeletal muscle $\cdot$ Neuromuscular junction $\cdot$ Spinal cord

\section{Introduction}

Spinal muscular atrophy (SMA) is an autosomal-recessive neuromuscular disorder representing the leading genetic cause of pediatric mortality in children younger than 2 years of age, with an incidence of 1 in 6000-10,000 live births and a carrier frequency of 1 in 35-50 [1,2]. The histopathological hallmark of SMA is the progressive loss of $\alpha$-motor neurons (MNs) in the spinal cord and lower brainstem, leading to weakness, paralysis, and subsequent atrophy of skeletal muscles. Clinically, the phenotypes of this disorder are heterogeneous, ranging from severe (type I) to mild (types III and IV), and patients are classified according to the age of disease onset and the severity of motor function.

In about $95 \%$ of cases, SMA is caused by homozygous deletion or inactivating mutations of the telomeric copy of $S M N 1$, located on the long arm of chromosome 5 [3]. This gene encodes the survival of motor neuron (SMN) protein, which is ubiquitously expressed and plays an essential role in mRNA splicing by regulating the assembly of small nuclear 
ribonucleic proteins and pre-mRNAs maturation [4, 5]. Levels of full-length SMN (FL-SMN) protein are dramatically reduced in SMA. Humans have a SMN1 paralog, called SMN2, also located on chromosome 5 but in a centromeric position. SMN2 differs from SMN1 in a C-to-T transition in exon 7. This leads to the alteration of the normal splicing and the inefficient production of full-length transcripts. Thus, about $85-90 \%$ of SMN2 transcripts lack exon 7 and produce a truncated and unstable protein (SMN $\Delta 7$ ), which is rapidly degraded and therefore not functional [6]. The remaining $(\sim 10-15 \%)$ transcripts derived from the SMN2 produce a FL-SMN protein, which is only able to compensate partially for the absence of SMN1. SMN2 copy number is inversely correlated with the severity of the disease $[7,8]$, and usually the most affected patients are those with fewer copies of SMN2.

The reason why reduced levels of the ubiquitous SMN protein lead to predominant alterations in the neuromuscular system is still unclear. It has been suggested that alterations in small nuclear ribonucleic protein assembly due to low SMN levels disturb the splicing of groups of genes that are crucial for $\mathrm{MN}$ function. Moreover, it is known that SMN plays important roles in motor axons and growth cones (reviewed in [9]), and a recent study [10] provided evidence of a presynaptic localization and interaction of this protein with the heterogeneous nuclear ribonucleoprotein $\mathrm{R}$ in motor axon terminals, suggesting that SMN could contribute to differentiation and maintenance of the neuromuscular junction (NMJ). In addition, SMN deficiency induces intrinsic defects in skeletal muscle that appear to contribute to motor disturbances observed in SMA ([11-16]; see also [17] for a review). Currently, there is no effective therapy for SMA except for palliative care, such as nutritional supplementation, and orthopedic and respiratory support, to improve quality of life. However, tremendous efforts have been made in the last decade to find useful strategies to halt the progression of neurodegeneration linked to this disease. Owing to the fact that all patients with SMA have at least 1 copy of $S M N 2$, most of the therapeutic strategies developed thus far are focused on increasing the synthesis of endogenous FL-SMN protein derived from SMN2 through different mechanisms. Some of these approaches have been used in mouse models of SMA and clinical trials, and include several histone deacetylase inhibitors, aminoglycosides, C5-quinazoline derivatives, $\beta$-adrenergic agonists, and chemical inhibitors of glycogen synthase kinase 3, among of other compounds [18-26]. Other treatments are based on the use of antisense oligonucleotides, which act on SMN2 splicing by restoring the inclusion of exon 7 in the final mRNA transcript with the subsequent enhancement of SMN expression, and on gene therapy through the insertion of the entire $S M N 1$ cDNA sequence by using viral vectors [21, 24, 27-32]. However, because MNs and skeletal muscles may require particular conditions to respond adequately to treatments designed to enhance SMN levels, SMNindependent strategies could also be very useful as adjunct treatments for SMA. These approaches are based on the use of neurotrophic factors and other neuroprotective and myotrophic compounds, and on stem cell therapies aimed at replacing MNs that are lost, and/or to confer MN protection through the delivery of neurotrophic molecules by transplanted cells (see $[19,20,26,27,33,34]$ for a review).

The potential role of physical exercise as an adjunct therapy in MN disorders has been studied in mouse models of amyotrophic lateral sclerosis and SMA [35-39]. Thus, regular exercise has been reported to improve motor function significantly, prolong lifespan, protect MNs from death, and attenuate muscular atrophy in mice mimicking SMA type II [38]. These beneficial effects of physical exercise appear to be related not only to the increase in exon 7-containing SMN transcripts, seen in the spinal cord of trained SMA animals [38], but also to the increased activation of $N$-methyl-D-aspartate (NMDA) subtype glutamate receptors, which is decreased in MNs of affected SMA mice [39]. On the other hand, NMDA receptor activation has been shown to enhance SMN levels in vitro [40].

Adenosine monophosphate-activated protein kinase (AMPK) is known to be activated in skeletal muscle during exercise and has an important role in the regulation of muscle metabolism, transcription, and phenotypic plasticity [41-45]. The synthetic AMPK agonist 5-aminoimidazole-4carboxamide-1- $\beta$-D-ribofuranoside (AICAR) is a potent stimulator of AMPK activity in skeletal muscle and, when administered chronically, has been reported to induce important plastic changes in muscle cells [46-49]. AICAR has also been demonstrated to increase training adaptation and enhance endurance in the absence of physical activity by acting as an exercise mimetic agent [50]. Pharmacological AMPK activation with AICAR has been assayed in the dystrophin-deficient $m d x$ mouse as a model of Duchenne muscular dystrophy. In this paradigm, chronic treatment with AICAR has been shown to ameliorate disease muscle phenotype and motor behavior [51-54].

In the present study, we investigated whether chronic treatment with AICAR is able to exert beneficial effects on lifespan, phenotype, motor abilities, and neuromuscular histopathology in a mouse model of severe SMA (the SMN $\Delta 7$ mouse), in a similar way as reported for physical exercise. We show here that AICAR induce relevant changes in SMN $\Delta 7$ mice by improving skeletal muscle atrophy, promoting the maturity of muscle fibers, and ameliorating some structural alterations found in NMJs. Although AICAR was also able to prevent the loss of glutamatergic excitatory synapses on MNs linked to the disease, this agent did not have any positive effects in mitigating MN loss or the microgial and astroglial reaction occurring in the spinal cord of SMN $\Delta 7$ mice. Moreover, AICAR did not elicit any significant improvement in survival and motor performance of diseased animals. 


\section{Materials and Methods}

\section{Mice and Pharmacological Experiments with AICAR}

The $S m n^{+/-} ; S M N 2^{+/+} ; S M N \Delta 7^{+/+}$[heterozygote knockouts for Smn, FVB.Cg-Tg( S M 2*delta 7$) 4299$ A h mb Tg(SMN2)89Ahmb Smn1tm1Msd/J, stock number 005025] mice were purchased from The Jackson Laboratory (Sacramento, CA, USA). Heterozygous female and male animals were crossed to obtain $S m n^{-/-} ; S M N 2^{+/+} ; S M N \Delta 7^{+/+}$(homozygote knockouts for $S m n$, hereafter referred to as SMN $\Delta 7$ ) and $S m n^{+/+} ; S M N 2^{+/+} ; S M N \Delta 7^{+/+}$(wild-type for Smn, hereafter referred to as WT) mice. SMN $\Delta 7$ mice exhibit a severe postnatal SMA phenotype, with overt symptomatology and histopathological changes in the spinal cord and skeletal muscles at 7-8 days after birth, and have a mean lifespan of about 2 weeks [55-57].

At postnatal day (P) 0, the feet of mice were marked with tattoo paste (Fine Science Tools GmbH, Heidelberg, Germany) for identification purposes, and a small piece of tail was cut for subsequent genotyping. The REDExtract-N-Amp Tissue PCR Kit (Sigma, St. Louis, MO, USA) was used for genomic DNA extraction and polymerase chain reaction setup. The primers used were as follows: WT forward 5' ctccgggatattgggattg 3', SMA reverse 5' ggtaacgccagggtttcc 3'; WT reverse 5' tttcttctggctgtgccttt 3'. Electrophoresis in $1 \%$ agarose gel and SYBR safe DNA stain (Molecular Probes, Eugene, OR, USA) was run at 100 V. Age-matched WT littermates of mutant animals were used as controls.

Animals were divided into 4 experimental groups: WT and $\mathrm{SMN} \Delta 7$ mice treated with AICAR $(500 \mathrm{mg} / \mathrm{kg} /$ day; Tocris Bioscience, Bristol, UK), and WT and SMN $\Delta 7$ mice treated with the corresponding volume of physiological saline solution (vehicle). AICAR or vehicle was administered, through subcutaneous interscapular injections, once a day in the morning from P1 until the end of the experimental time frame. At selected times, mice were anesthetized with an intraperitoneal injection of pentobarbital ( $2 \%, 1 \mathrm{ml} / 10 \mathrm{~g}$ body weight) and sacrificed by quick decapitation. Tissue samples were rapidly dissected, removed, and subsequently processed as described below. All the experimental procedures were carried out in agreement with the guidelines of the committee for animal care and use of the University of Lleida, approved by the Generalitat de Catalunya.

\section{Behavioral Phenotype Testing}

To evaluate disease progression, mice were weighed daily in the morning and, subsequently, carefully examined in order to determine the presence of specific signs and/or symptoms of disease. Thereafter, the righting reflex and the hindlimb suspension test ("tube test") were conducted by the same investigator (blind to the experimental condition) to assess the motor abilities of mice. These tests were scored following the guidelines described elsewhere [58]. In brief, for the righting reflex, mice were placed on their backs on a flat surface, and the time necessary to reposition themselves dorsal side up was assessed over a period of $30 \mathrm{~s}$. For the tube test, each mouse was suspended head down from its hindlimbs in a 50-ml plastic centrifuge tube containing a piece of cotton at the bottom to protect the animal when they fell. For this test, 2 parameters were evaluated: the latency (in seconds) to fall from the edge of the tube, and the positioning of the legs and tail. For the latter parameter, posture was scored from 4 to 0 as follows: 4 , hindlimbs were normally separated and tail raised; 3, weakness was apparent and hindlimbs were closer together but only occasionally touched each other; 2 , hindlimbs were close to each other and frequently touching; 1, hindlimbs were almost always in a clasp position with the tail raised; 0 , hindlimbs were in constant clasping and the tail lowered; a score of 1 was given when a mouse failed to hold on to the tube. For each test, every mouse was examined twice, with a $15-\mathrm{min}$ recovery period between trials; the average of values obtained in the 2 independent tests was then determined for the final score.

\section{Muscle and Spinal Cord Histology and MN Counts}

Tibialis anterior (TA) and intercostalis (IC) muscles, and spinal cords were rapidly dissected and processed for histological analysis and $\mathrm{MN}$ counting.

TA muscles were weighed, fixed in $4 \%$ paraformaldehyde in $0.1 \mathrm{M}$ phosphate buffer (PB) at $\mathrm{pH} 7.4$ for $2 \mathrm{~h}$, cryoprotected with $30 \%$ sucrose in $0.1 \mathrm{M}$ in PB, embedded in Tissue Freezing Medium (Triangle Biomedical Sciences, Durham, NC, USA), and frozen. Multiple cryostat transverse sections (16- $\mu \mathrm{m}$ thick) were obtained from the midbelly of the muscle. Sections were subsequently stained with hematoxylin and eosin (H\&E).

For MN counts, spinal cords were fixed in Bouin's solution and embedded in paraffin. Serial transverse sections $(12-\mu \mathrm{m}$ thick), obtained through the entire lumbar segment, were stained with H\&E. The apparently healthy MNs present in the ventral horn were identified by their size and shape, and counted blindly on one side of every 10th section according to previous described procedures [59, 60]. Briefly, only MNs with a large nucleus, a visible clump of nuclear material, and a substantial cytoplasm were counted. The total number of MNs per ventral horn was obtained by multiplying the number of counted cells by 10 .

\section{Immunocytochemistry and Imaging}

Unless otherwise indicated, for immunocytochemical studies, TA and IC muscles and lumbar spinal cords were fixed by immersion in $4 \%$ paraformaldehyde in $0.1 \mathrm{M} \mathrm{PB}, \mathrm{pH} 7.4$, either for $2 \mathrm{~h}$ (in the case of TA and IC muscles) or overnight (in the case of spinal cords), and cryoprotected. Tissue samples were embedded in Tissue Freezing Medium (Triangle Biomedical Sciences) and frozen. Longitudinal (for TA and IC muscles) or transverse 
(for spinal cord) serial cryostat sections (16- $\mu \mathrm{m}$ thick) were obtained and stored at $-80{ }^{\circ} \mathrm{C}$. Immunocytochemical analysis of muscle fiber type composition was performed on unfixed TA muscles. For this, muscles were embedded in tragacanth gum, snap-frozen in liquid $\mathrm{N}_{2}$-cooled isopentane, and sectioned transversely $(10-\mu \mathrm{m}$ thick) on a cryostat.

Sections were sequentially rinsed in phosphate-buffered saline containing $0.1 \%$ Triton X-100 for $30 \mathrm{~min}$, blocked in normal goat serum, and incubated with the selected primary antibody overnight. The following primary antibodies were used: rabbit polyclonal antivesicular glutamate transporter 1 (VGluT1) (diluted 1:1000; catalog no. 135 302, Synaptic Systems, Gottingen, Germany); rabbit polyclonal anti-ionized calcium-binding adaptor molecule 1 (IBA1) (diluted 1:500; catalog no. 019-19741, Wako Pure Chemical Industries Ltd, Osaka, Japan); chicken polyclonal antiglial fibrillary acidic protein (GFAP) (diluted 1:1000; catalog no. Ab4674, Abcam, Cambridge, UK); rabbit polyclonal anti-NMDA receptor 2A subunit (NR2A) (diluted 1:1000; catalog no. AB1555P, Chemicon International Inc., Billerica, MA, USA); rabbit polyclonal anticalcitonin gene-related peptide (CGRP) (diluted 1:1000; catalog no. C8198, Sigma); chicken polyclonal antineurofilament $68 \mathrm{KDa}(\mathrm{NF})$ (diluted 1:1000; catalog no. ab72997, Abcam); mouse monoclonal antisynaptic vesicle protein 2 (SV2) [diluted 1:1000; catalog no. SV2, Developmental Studies Hybridoma Bank (DSHB), Iowa City, IA, USA]; mouse monoclonal antimyosin heavy chain (MyHC) I (diluted 1:5; catalog no. A4-840, DSHB); mouse monoclonal anti-MyHC IIA (diluted 1:20; catalog no. SC-71, DSHB); and mouse monoclonal anti-MyHC IIB (diluted 1:20; catalog no. BF-F3, DSHB). After incubation with the primary antibody, sections where washed and incubated at room temperature (RT) for $1 \mathrm{~h}$ with the appropriate secondary fluorescent antibodies: Dylight 488 donkey antirabbit IgG (diluted 1:500; Jackson ImmunoResearch Laboratories, West Grove, PA, USA); Cy3 donkey antichicken or antirabbit (diluted 1:500; Jackson ImmunoResearch Laboratories); Alexa Fluor 488 goat antichicken IgG (diluted 1:500; Molecular Probes). Sections were also labeled with 4',6-diamidino-2-phenylindole dihydrochloride (50 ng/ml; Molecular Probes) for DNA staining. Muscle sections were incubated with Alexa Fluor 488- or Alexa Fluor 647-labeled $\alpha$-bungarotoxin ( $\alpha$-Bgtx) (diluted 1:500; Molecular Probes) to identify postsynaptic acetylcholine receptors. Spinal cord sections were counterstained with Neuro Trace 500/525 or 530/615 (green or red, respectively) fluorescent Nissl stain (Molecular Probes) to identify ventral horn MNs. After washing, slides were coverslipped by using Vectashield (Vector Laboratories, Burlingame, CA, USA) or Mowiol (Calbiochem, San Diego, CA, USA). Slides were examined with an Olympus BX51 epifluorescence microscope (Olympus, Hamburg, Germany) equipped with a DP30BW camera or FluoView 500 or FluoView 1000 Olympus laser scanning confocal microscopes. For comparisons, slides from different animals and experimental conditions were processed in parallel for immunocytochemistry and subsequent imaging. The same scanning parameters were used for the acquisition of images corresponding to different experimental groups. For spinal cord immunocytochemical studies, digital images of the entire lumbar region were obtained from every 30 th section.

\section{Image and Morphometric Analysis}

Myofiber number counts and size measurements were performed in bright field digital images taken from TA muscle cross-sections stained with H\&E. Images were subsequently processed using ImageJ software (National Institutes of Health, Bethesda, MD, USA).

The cytoarchitecture of NMJs was analyzed in longitudinal immunostained sections of TA and IC muscles. Z-stack optical sections $(1-\mu \mathrm{m}$ thick) were obtained and projected to reconstruct NMJs using the microscope software. Five to nine sections per muscle were analyzed for each animal, and 75-100 NMJs from randomly selected visual fields were evaluated per experimental condition. NMJ size was assessed by determining the area of the $\alpha$-Bgtx-labeled postsynaptic site, manually outlined, using ImageJ. A NMJ was considered to be denervated when the percentage of $\alpha$-Bgtx-labeled postsynaptic surface covered by SV2-stained presynaptic terminal was $<15 \%$. Single or poly-innervation was estimated by counting the number of preterminal axons (revealed by NF immunostaining) entering a single postsynaptic site: a NMJ was considered to be polyinnervated when $\geq 2$ axons were seen converging on the postsynaptic site. Based on the structural appearance of each postsynaptic site, the degree of maturity of NMJs was classified as follows: plaque, folds, perforations, or secondary structure (ranging from immature to mature) according to previously described criteria [61]. NF was considered to accumulate in the presynaptic sites when the terminal portion of axons exhibited a swollen morphology and was strongly stained with the anti-NF antibody. Terminal sprouting was quantified by counting the number of NF-stained axonal processes coming from a nerve terminal that escaped from an $\alpha$-Bgtx-stained postsynaptic site. The number of sprouts counted per NMJ was referred to as a percentage of the total number of NMJs examined per muscle. CGRP immunostaining in NMJs was scored, by a blinded observer, according to the fluorescence intensity and classified as follows: positive, mild (low signal but distinguishable from the background), or negative. Any NMJ that was difficult to analyze owing to its location and/or orientation was excluded from the analysis.

Levels of GFAP and IBA1 immunoreactivity in the spinal cord were quantified by digital image analysis using the Visilog 6.3 software (Noesis, Orsay, France). The number of VGluT1 immumunoreactive synaptic boutons on MN somata was counted manually on the screen; only boutons in close contact 
with MNs showing a large nucleus, a visible nucleolus, and a healthy appearance were included in the counts, which were then normalized to the perimeter of $\mathrm{MN}$ soma.

\section{Western Blotting}

Frozen spinal cords and hindlimb muscles were fragmented and homogenized with blending buffer $1 \times \mathrm{SR}(2 \%$ sodium dodecyl sulfate and $125 \mathrm{mM}$ Tris-HCl, $\mathrm{pH}$ 6.8) supplemented with protease inhibitor (Ref. P8340; Sigma) and PhosSTOP (Roche, Laval, Canada). Homogenized samples were heated to $100^{\circ} \mathrm{C}$ for $5 \mathrm{~min}$ and centrifuged at $13,000 \mathrm{~g}$ for $5 \mathrm{~min}$. The protein concentrations of supernatants were determined by BIO-RAD Micro DC protein assay (BIO-RAD Laboratories Inc., Hercules, CA, USA). Loading buffer $4 \times$ SS (20 \% sucrose and $0.05 \%$ bromophenol blue, $0.1 \%$ sodium azide) containing 5-10\% $\beta$-mercaptoethanol (Sigma) and 15$50 \mu \mathrm{g}$ protein were loaded in a $10 \%$ or $15 \%$ polyacrylamide electrophoresis gel. Proteins were electrotransferred to polyvinyldifluoride membranes (ImmobilonTM-P, Millipore, Bradford, MA, USA) in Tris-glycine-methanol-buffered solution. Membranes were blocked with $5 \%$ dried skimmed milk in $0.1 \%$ Tween 20 and Tris-buffered saline $\mathrm{pH} 8$ (TBST) for $1 \mathrm{~h}$ at RT, and then extensively washed in TBST. Immunodetection was performed by incubating the membranes overnight at $4{ }^{\circ} \mathrm{C}$ with the following antibodies: rabbit polyclonal antiNR2A (diluted 1:1000; catalog no. AB1555P, Chemicon International Inc.); rabbit polyclonal anti-AMPK $\alpha$ (diluted 1:1000; catalog no. 2532, Cell Signaling, Beverley, MA, USA); rabbit polyclonal antiphospho-AMPK $\alpha$ (Thr172) (diluted 1:1000; catalog no. 2531, Cell Signaling); rabbit polyclonal antiperoxisome proliferator-activated receptor- $\gamma$ coactivator $1(\mathrm{H} 300)(\mathrm{PGC}-1 \alpha)$ (diluted 1:200; catalog no. SC-13067, Santa Cruz Biotechnology, Inc., Santa Cruz, CA, USA); mouse monoclonal anti-SMN (diluted 1:1000; catalog no. 610647, BD Biosciences, San Jose, CA, USA); mouse monoclonal anti- $\beta$-actin (diluted 1:5000; catalog no. A54441, Sigma); mouse monoclonal anti- $\alpha$-tubulin (diluted 1:5000; catalog no. T5168, Sigma); and mouse monoclonal antiglyceraldehyde 3-phosphatase dehydrogenase (6C5); (diluted 1:10,000; catalog no. ab8245, Abcam). The antibodies against $\beta$-actin and $\alpha$-tubulin or glyceraldehyde 3-phosphatase dehydrogenase were used for loading controls in blots of spinal cord and muscle extracts, respectively. The membranes were washed in TBST, incubated with the appropriate peroxidase-conjugated secondary antibodies (1:5000; Amersham Biosciences, Little Chalfont, UK) for $60 \mathrm{~min}$ at RT, washed in TBST, and visualized using the ECL Prime Western Blotting Detection Reagent detection kit (GE Healthcare, Little Chalfont, UK), as described by the manufacturer. The quantification of band densities was performed by using Chemi-Doc MP Imaging System (BIO-RAD Laboratories Inc.).

\section{Statistical Analysis}

All data are expressed as means \pm SEM. The statistical analysis was assessed by either one-way analysis of variance, followed by post-hoc Bonferroni's test, or Student's $t$ test. Differences were considered to be statistically significant if $p \leq 0.05$.

\section{Results}

\section{Effects of AICAR Treatment on AMPK Phosphorylation and PGC-1 $\alpha$ Content in the Skeletal Muscle of SMN $\Delta 7$ Mice}

We first examined the effect of AICAR administration on AMPK activity, evaluated by phosphorylation, in the skeletal muscle of WT and SMN $\Delta 7$ mice in vivo. For this, either AICAR $(500 \mathrm{mg} / \mathrm{kg})$ or saline was injected subcutaneously, once daily, to animals from P1 to P8, and hindlimb muscles were collected on P8, $3 \mathrm{~h}$ after the last injection. The AICAR dosage, and regimen and route of administration used here were the same as reported by others in previous studies [51, 62]. Western blot analysis of muscle extracts showed no significant changes in total AMPK protein levels after AICAR treatment in both WT and SMN $\Delta 7$ animals (Fig. 1A, B). However, AMPK phosphorylation, examined with an antiphospho-AMPK (Thr172) antibody, displayed a moderate ( $\sim$.6-fold) increase in muscles of AICAR-treated WT mice compared with those of WT animals injected with saline (Fig. 1A, C). Strikingly, we noticed that muscles of salinetreated SMN $\Delta 7$ animals showed significantly greater levels ( $\sim 1.6$-fold increase; $p<0.05$ ) of phosphorylated AMPK compared with the saline-WT condition. It is important to note that we also found a similar increase in phosphorylated AMPK expression in muscles of $\mathrm{Smn}^{-/} ; \mathrm{SMN}^{+/+}$mice, a more severe model of the disease mimicking type I SMA (see supplementary Fig. S1). This elevated AMPK phosphorylation in muscles of diseased animals was not increased further after AICAR treatment; indeed, we observed a modest, but not significant, decrease in phosphorylated AMPK in SMN $\Delta 7$ muscles of animals treated with AICAR in relation to those injected with saline $(p>0.05)$ (Fig. 1A, C). The phosphorylation status of AMPK, based on the ratio of phosphorylated AMPK relative to total AMPK levels, showed a similar profile to that of phosphorylated AMPK (Fig. 1D). Overall, these results indicate that basal AMPK activity in skeletal muscles is significantly elevated in SMA animals. Moreover, chronic treatment with AICAR modestly activates AMPK in skeletal muscles of healthy mice in vivo but is not able to modify significantly AMPK phosphorylation levels found in the course of the disease.

PGC- $1 \alpha$ is a transcriptional coactivator that appears to play an important role in the oxidative metabolism of skeletal 
a

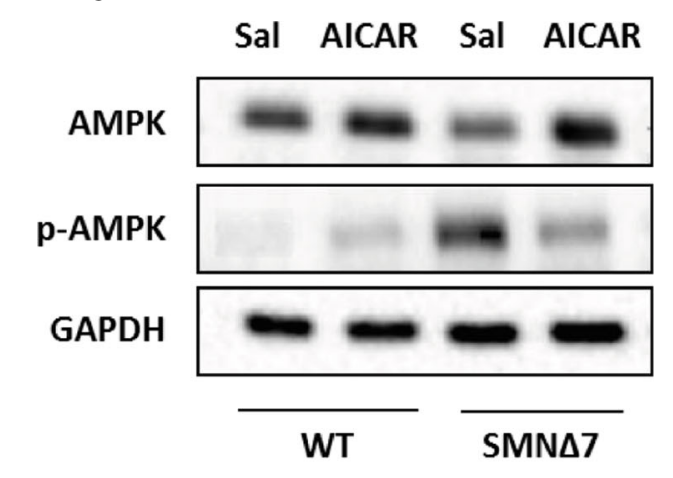

c

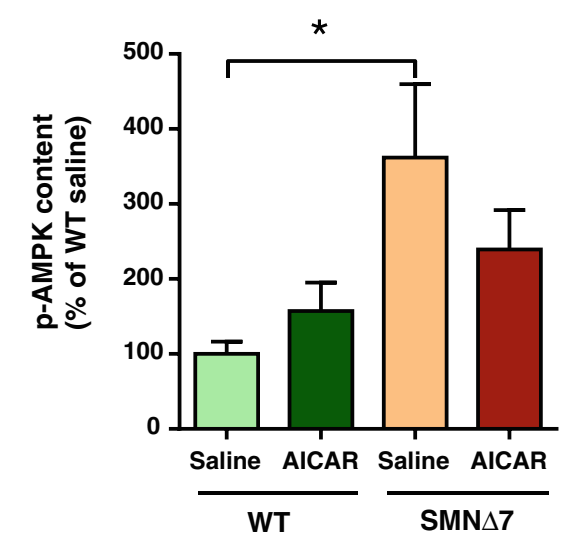

e

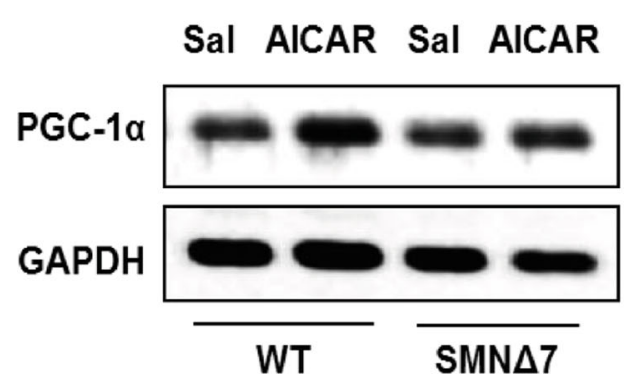

b

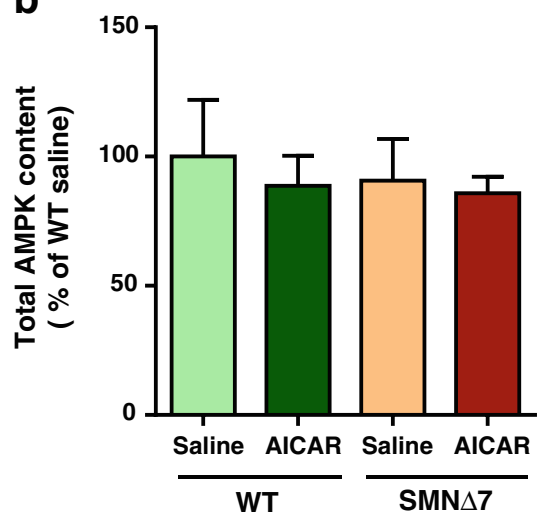

d

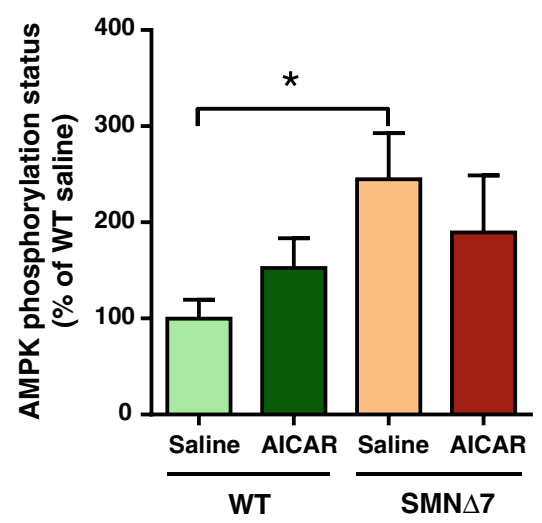

f

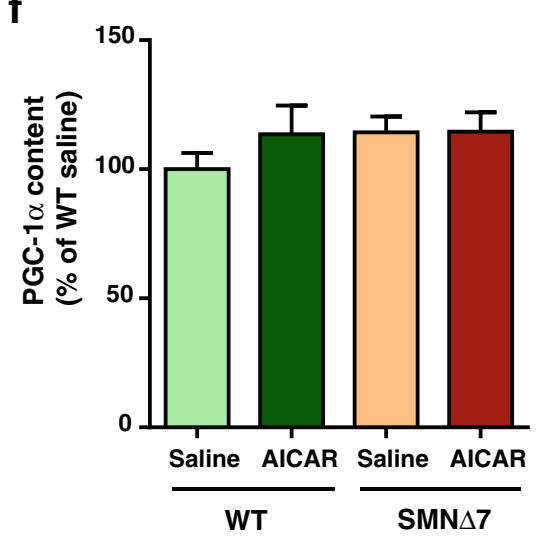

Fig. 1 Effects of chronic 5-aminoimidazole-4-carboxamide-1- $\beta$-Dribofuranoside (AICAR) treatment on total and phosphorylated adenosine monophosphate-activated protein kinase (AMPK) protein levels, and peroxisome proliferator-activated receptor- $\gamma$ coactivator 1 (PGC-1 $\alpha)$ content in the skeletal muscle of wildtype (WT) and $S m n^{-/-} ; S M N 2^{+/+} ; S M N \Delta 7^{+/+}(\mathrm{SMN} \Delta 7)$ mice. Muscles from hindlimbs were collected on postnatal day 8 after daily treatment with either $500 \mathrm{mg} / \mathrm{kg}$ AICAR or vehicle [saline solution (Sal)]. (A) Representative Western blots for total AMPK, phosphorylated AMPK (p-AMPK) and glyceraldehyde-3phosphate dehydrogenase (GAPDH; as loading control). (B-D) Quantification of changes in total (B) AMPK and (C) p-AMPK content, determined by densitometry, and (D) the phosphorylation status of the kinase in basal conditions (animals injected with

saline) and after AICAR treatment. The phosphorylation status of AMPK is represented as the ratio of the phosphorylated form relative to total AMPK. Data in all graphs are expressed as the percentage of change in relation to the levels found in the salineWT group. Bars represent the values (mean \pm SEM) of 8 mice per experimental condition from 3 independent experiments. (E) Representative Western blots of PGC- $1 \alpha$ protein and GAPDH (as loading control). (F) Densitometric analysis of changes in PGC $-1 \alpha$ content in basal conditions (animals injected with saline) and after AICAR treatment. Bars represent the values (mean $\pm \mathrm{SEM})$ of 5-6 mice per experimental condition from 2 independent experiments. ${ }^{*} p<0.05$, one-way analysis of variance (Bonferroni's post-hoc test) 
muscle by stimulating mitochondrial biogenesis and oxidative enzymes [63, 64]. Exercise has been reported to induce PGC$1 \alpha$ expression in both rodents and humans [65-67]. It is known that AMPK is able to phosphorylate directly PGC$1 \alpha$ protein resulting in the induction of the PGC- $1 \alpha$ promoter [68]. PGC- $1 \alpha$ content in muscle increases after chronic pharmacological AMPK stimulation by AICAR [50]. For this reason, we next explored whether AICAR treatment enhanced PGC- $1 \alpha$ levels in skeletal muscles of SMN $\Delta 7$ mice. Western blot examination of muscle extracts of AICAR-treated WT mice showed a slight, but not significant, increase ( $\sim 1.2$-fold) in PGC- $1 \alpha$ in relation to levels found in saline-injected WT animals. A similar modest increase in PGC- $1 \alpha$ content was observed in muscles of saline- and AICAR-injected SMN $\Delta 7$ mice. These results indicate that, in our experimental model, AICAR treatment has a minor influence on the levels PGC- $1 \alpha$ protein in skeletal muscle.

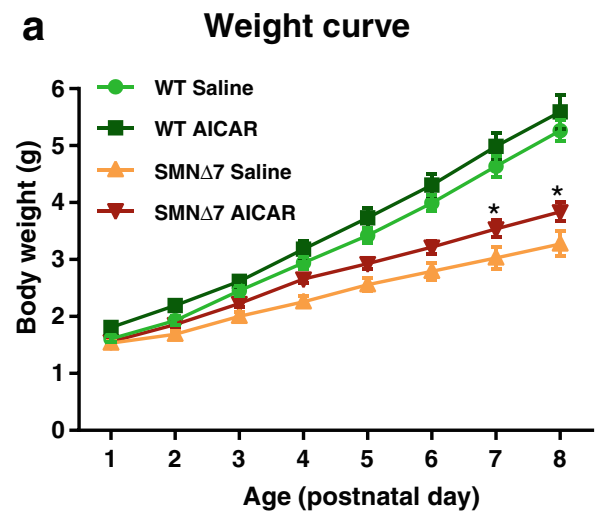

C Hindlimb suspension (tube) test

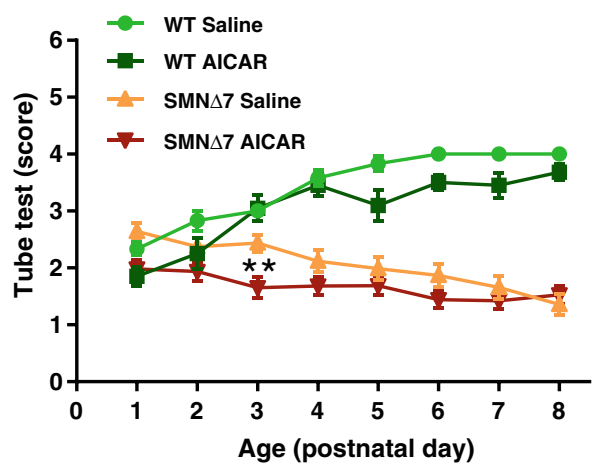

Fig 2 Chronic treatment with 5-aminoimidazole-4-carboxamide-1- $\beta$-Dribofuranoside (AICAR) modestly ameliorates the deficiency in body weight gain of $\mathrm{Smn}^{-1-} ; \mathrm{SMN2}^{+/+} ; S M N \Delta 7^{+/+}(\mathrm{SMN} \Delta 7)$ mice but does not improve their motor phenotype. (A) Body weight of wild-type (WT) and SMN $\Delta 7$ mice treated with either saline or AICAR. Note that compared with saline-injected SMN $\Delta 7$ mice, mutant animals treated with AICAR exhibit higher weights as early as postnatal day (P) 4; however, these differences are only significant at P7 and P8 $\left({ }^{*} p<0.05\right.$ vs $\mathrm{SMN} \Delta 7$ saline). (B, C) Motor behavior assessment by using (B) righting reflex and $(\mathrm{C})$ hindlimb suspension test; except for the tube test at $\mathrm{P} 3$, in which SMN $\Delta 7$ mice treated with AICAR have significantly

\section{Chronic Treatment with AICAR did not Improve Motor Abilities and Lifespan of SMN $\Delta 7$ Mice}

The progression of SMA in mouse models results in a gradual weight loss and impaired motor performance [56]. As expected, SMN $\Delta 7$ mice treated with saline showed a lower growth-related gain of body weight in relation to saline-injected WT animals. SMN $\Delta 7$ mice chronically treated with AICAR displayed a modest improvement in body weight gain. Although this change was already observed at $\mathrm{P} 4$, it was statistically significant at P7 and P8 $(p<0.05)$. No significant differences in body weight were found at any experimental time point when WT mice treated with either AICAR or saline were compared (Fig. 2A).

Righting reflex and the tube test were used as assessments of the muscle strength and motor coordination. Both saline- and AICAR-treated SMN $\Delta 7$ mice showed similar times to right themselves on all 4 paws, with significant difficulties in righting

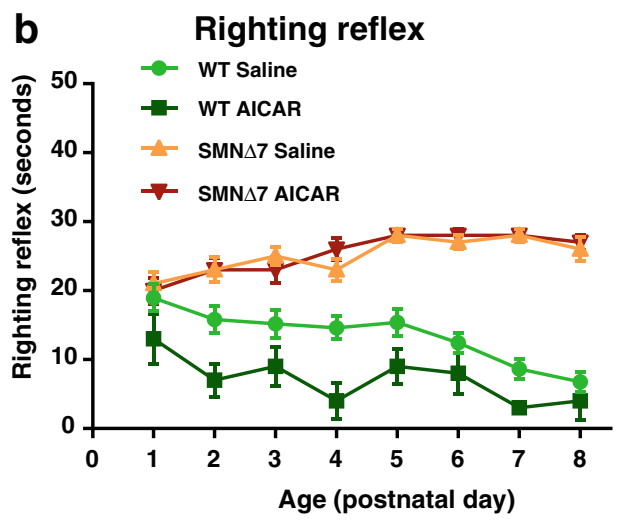

d

\section{Survival curve}

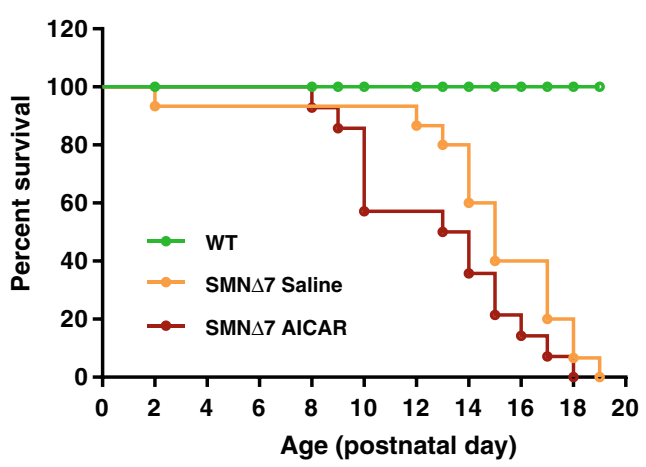

lower scores than those injected with saline, no significant differences in righting reflex or tube test performance were observed between these 2 groups at any of the ages examined ( ${ }^{* * *} p<0.001 v s$ SMN $\Delta 7$ saline). In all graphs, values are shown as mean \pm SEM, and one-way analysis of variance (Bonferroni's post-hoc test) was used for statistical analysis ( $n=15-28$ mice per experimental group). (D) Kaplan-Meier survival curve for saline-injected and AICAR-treated SMN $\Delta 7$ mice, and WT littermates. Saline- and AICAR-treated SMN $\Delta 7$ mice did not show significant differences in the mean survival (14.6 \pm 1 and $12.8 \pm 0.8$ days, respectively; $p>0.05$, Student's $t$ test, $n=14-15$ per experimental condition 
in comparison with saline- and AICAR-treated WT mice (Fig. 2B). Although on most of the days tested AICARinjected WT animals showed shorter times to right than salineinjected WT mice, the differences were not statistically significant. In relation to WT animals, both saline and AICAR-treated SMN $\Delta 7$ mice had short latencies to fall and worse scores when assessed in the tube test (Fig. 2C). At P3, significantly lower tube test scores were observed in AICAR-treated SMN $\Delta 7$ mice versus diseased animals injected with saline; however, this decline was not maintained during the remaining days studied.

To determine whether AICAR treatment is able to prolong the lifespan of SMN $\Delta 7$ mice, survival was monitored in a group of animals injected daily with either the AMPK agonist or saline from P1 until death. The Kaplan-Meier survival curve is shown in Fig. 2D. AICAR administration did not elicit any significant effect on the lifespan of diseased animals (mean survival: SMN $\Delta 7$-saline $14.6 \pm 1$ days, $n=15$; $\mathrm{SMN} \Delta 7$-AICAR $12.8 \pm 0.8$ days, $n=14 ; p>0.05$ ). Moreover, in this group of SMN $\Delta 7$ mice that received AICAR until the end stages of disease, no significant improvements in motor performance were found, and the enhancement in body weight was only maintained until P10 (data not shown).

\section{Chronic Treatment with AICAR Reduced Muscular Atrophy Associated with SMA}

To determine whether AICAR treatment is able to improve muscle pathology linked to SMA, the phenotype of the TA muscle was examined. As expected, TA muscle weights in saline-treated SMN $\Delta 7$ mice were significantly lower $(\sim 40 \%$ reduction; $p<0.01$ ) than those in WT animals (Fig. 3A). An increase $(\sim 15 \%)$ in muscle weight was observed in AICARtreated WT mice in relation to saline-injected WT littermates; however, this change was not statistically significant. Interestingly, chronic AICAR treatment significantly increased $(\sim 27 \%)$ the weight of TA muscle in SMN $\Delta 7$ mice, even though this increase did not reach the average weight values observed in WT animals treated with saline (Fig. 3A).

In concordance with previous reports [69], analysis of crosssections revealed that TA muscles of saline-treated SMN $\Delta 7$ animals had $\sim 19 \%$ fewer fibers than those of saline-injected WT littermates; however, probably because of the small sample size, these differences were not statistically significant. AICAR treatment resulted in a small, nonsignificant, increase $(\sim 1.3$-fold $)$ in the number of TA myofibers in SMN $\Delta 7$ mice (Fig. 3B).

As expected, TA muscle fibers of saline-treated SMN $\Delta 7$ mice showed a smaller cross-sectional area ( $\sim 32 \%$ reduction) compared with saline-treated WT littermates. In concordance with the changes in muscle weight induced by chronic AICAR treatment, we found a significant increase in the size of TA muscle fibers in both SMN $\Delta 7$ and WT mice injected with the AMPK agonist (Fig. $3 \mathrm{C}-\mathrm{G}$ ). The relative frequency distribution of myofiber areas demonstrated a shift to larger caliber fibers in TA muscle of both WT and SMN $\Delta 7$ animals treated with AICAR (Fig. 3H, I). Thus, in the absence of exercise training, chronic AICAR administration increases the size of myofibers and hence the mass of skeletal muscles of both normal and SMN $\Delta 7$ mice. In the latter, AICAR appears to be particularly beneficial, as it is able to limit the degree of muscular atrophy that results from the impaired postnatal myofiber growth linked to SMA $[13,69,70]$.

It has been reported that muscle fibers of SMN $\Delta 7$ mice, in addition to their reduced size, are less mature than those of normal animals [69, 70]; this results in changes in the fiber type composition of atrophied muscles. Accordingly, we next examined whether AICAR can modulate the alterations in the myofiber type profile observed in SMA. Specific MyHC isoforms are expressed in different types of muscular fibers [71]. Hence, different isoform-specific anti-MyHC (I, IIA, and IIB) antibodies were used to analyze the fiber type composition of WT and SMN $\Delta 7$ TA muscles (Fig. 3J-N). The pattern of fiber type composition in the TA muscle of saline-treated WT mice was similar to that previously described [72], with a very low proportion of type IA and IIA fibers and a predominance of type IIB fibers. Interestingly, in relation to saline-treated WT animals, TA muscles from saline-injected $\mathrm{SMN} \Delta 7$ mice showed significantly higher numbers of type I and a lower (although not significant) proportion of type IIB fibers. AICAR administration significantly increased the percentage of type I fibers in both WT and SMN $\Delta 7$ TA muscles with no apparent changes in type IIA and type IIB fibers. Thus, the TA muscle (a predominantly fast muscle type) of diseased animals exhibited a change in myosin expression profile with an abnormal increase in the abundance of type I (slow, oxidative) myofibers that was accentuated by AICAR treatment.

\section{AICAR Partially Restores NMJ Defects in SMN $\Delta 7$ Mice}

We next asked whether AICAR was able to modify structural abnormalities found in NMJs of SMN $\Delta 7$ mice. For this, muscle cryostat sections were double immunostained with antibodies against NF and SV2 to label axons and nerve terminals, respectively, and co-stained with $\alpha$-Bgtx to identify postsynaptic acetylcholine receptors. Denervation of the skeletal muscle has been described as a histopathological feature of SMA in humans that is recapitulated in distinct mouse models of the disease $[13,61,73]$. Denervation in the SMN $\Delta 7$ mouse model has been reported to occur in some specific muscles (i.e., in those from head and trunk), with only discrete changes in others (particularly in those located in hindlimbs) that appear to be fully innervated despite disease progression [73, 74]. In fact, we found a nonsignificant increase in the number of denervated NMJs in TA muscle of SMN $\Delta 7$ mice at P8, the age examined in the present study [WT $9.6 \% \pm 1.2 \%$, SMN $\Delta 712.3 \% \pm 1.6 \%(p>0.05), 78$ and 94 NMJs analyzed from 3-4 animals per condition]. For this reason we used the 
a

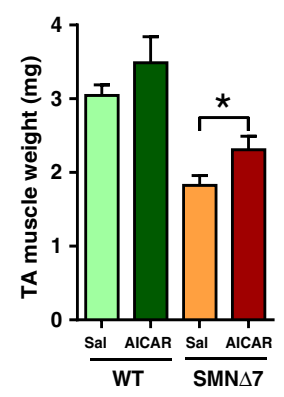

h
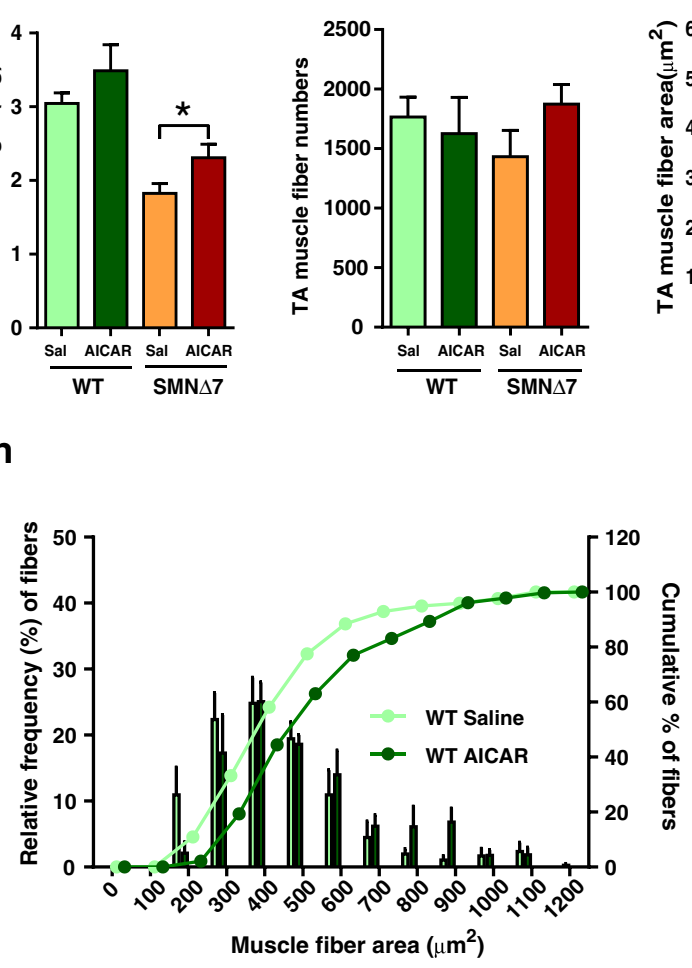
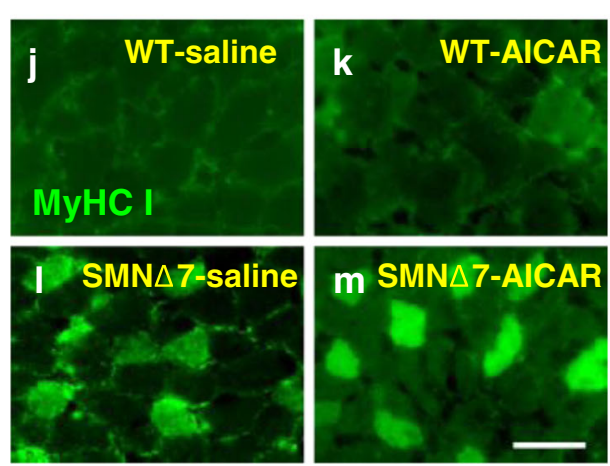

Fig. 3 Effects of chronic 5-aminoimidazole-4-carboxamide-1- $\beta$-Dribofuranoside (AICAR; $500 \mathrm{mg} / \mathrm{kg} /$ day) administration on tibialis anterior (TA) muscle of $S m n^{-/-} ; S M N 2^{+/+} ; S M N \Delta 7^{+/+}$(SMN $\left.\Delta 7\right)$ mice. (A) Weight of TA muscles from postnatal day (P) 8 WT and SMN $\Delta 7$ animals treated with either saline (Sal) or AICAR $\left({ }^{*} p<0.05 ; n=8-11\right.$ muscles per experimental condition). (B) Number of fibers in the TA muscle of P8 animals from the 4 experimental groups. There is a small, but not significant, reduction in the number of myofibers in saline-injected SMN $\Delta 7$ mice compared with saline-treated wild-type (WT) littermates; AICAR treatment increases fiber counts in muscles of mutant mice; however, the difference does not reach statistical significance ( $n=4$ muscles from distinct animals per group). (c) Cross-sectional area of muscle fibers in the TA muscle from P8 WT and diseased mice after treatments $(* * p<0.001 ; n=200-300$ fibers of TA muscles obtained from 4-5 mice per condition). (DG) Representative micrographs of TA muscle cryosections, stained with hematoxylin and eosin, from either saline- or AICAR-treated WT and SMN $\Delta 7$ mice. (H, I) Relative frequency of fiber sizes, expressed as a percentage of the total number of fibers; in TA
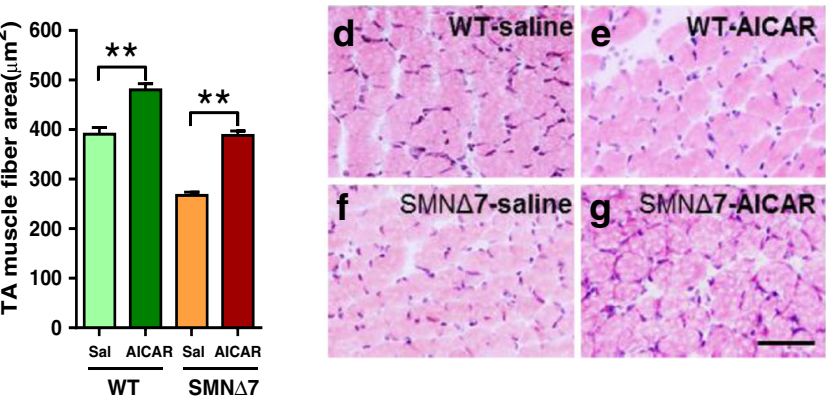

i
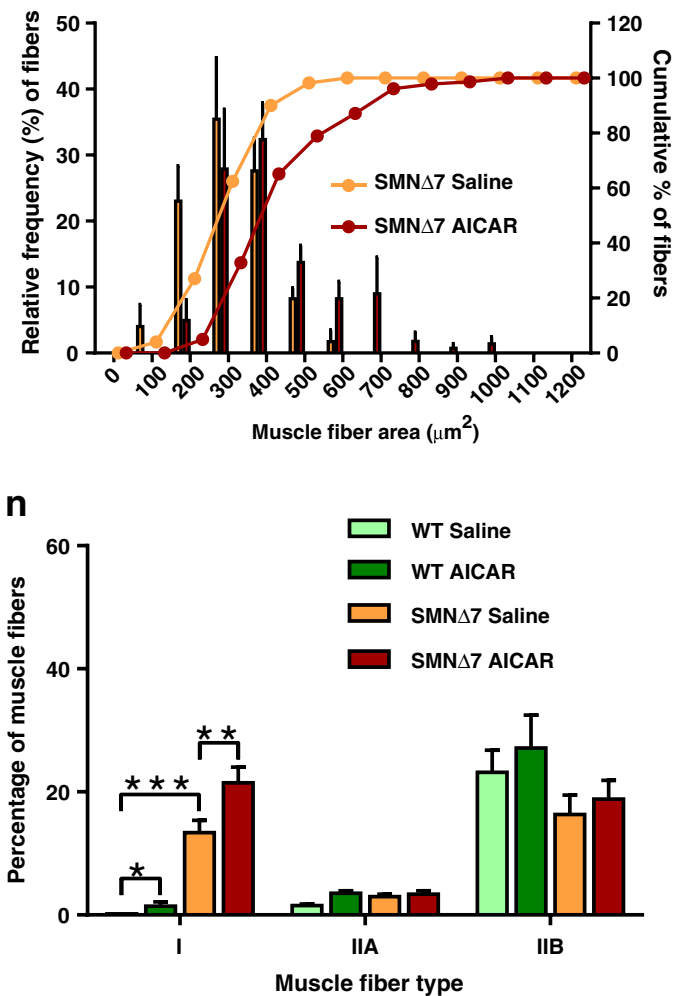

muscles from (H) WT and (I) SMN $\Delta 7$ mice subjected to saline or AICAR treatment ( $n=4-5$ mice per condition); the cumulative percentages of muscle fibers in each experimental group are also shown in the same graph. (J-M) Representative images of myosin heavy chain (MyHC) I immunostaining in TA muscles of $(\mathrm{J}, \mathrm{K})$ WT and (L, M) SMN $\Delta 7$ mice treated with either (J, L) saline or (K, M) AICAR. (N) Graphical summary of percentage of MyHC I-, MyHC IIA-, and MyHC IIB-immunostained fibers (type I, IIA, and IIB myofibers, respectively) in TA muscles of P8 WT and SMN $\Delta 7$ mice after AICAR treatment. Note that muscles from WT mice treated with AICAR display a moderate, but significant, increase in slow, MyHC I, fibers; compared to WT animals, muscles from saline-injected $\mathrm{SMN} \Delta 7$ animals show a marked elevation in the number of MyHC I fibers that is even higher after AICAR treatment $\left({ }^{*} p<0.05, * * p<0.01, * * * p<0.001\right.$, $n=20$ sections of TA muscles from $4-5$ mice per experimental condition). In all graphs, values are shown as mean \pm SEM, and one-way analysis of variance (Bonferroni's post-hoc test) was used for statistical analysis. Scale bar $=50 \mu \mathrm{m}$ in (G) (applies to $\mathrm{D}-\mathrm{G}$ ) and $25 \mu \mathrm{m}$ in (M) (applies to $\mathrm{J}-\mathrm{M}$ ) 
IC muscle, which has an important role in maintaining respiratory function and is clinically relevant in SMA. We know from previous studies that IC muscle displays important molecular and structural NMJ defects in SMA mice [13, 61]. In relation to P8 saline-treated WT animals, IC muscles of $\mathrm{SMN} \Delta 7$ mice treated with vehicle displayed a $\sim 1.5$-fold increase [WT-saline $25.0 \% \pm 1.1 \%$, SMN $\Delta 7$-saline $39.7 \% \pm$ $2.1 \%, n=97$ and $101 \mathrm{NMJs}$, respectively, from 3-4 mice per condition $(p<0.001)]$ in the number of denervated NMJs. AICAR treatment significantly prevented SMN $\Delta 7$ IC muscle denervation $(\sim 1.2$-fold reduction $v$ s. $\operatorname{SMN} \Delta 7$-saline; $p<0.05)$ linked to the disease (Fig. 4A-E").

It is known that NMJs of SMA mice display structural changes indicative of delayed synaptic maturation [61, 70, 75]. We found that, in the IC muscles of saline-injected SMN $\Delta 7$ mice, $\sim 40.5 \%$ of NMJs were innervated polyneuronally compared with $\sim 8 \%$ polyneuronal innervation observed in saline-treated WT littermates $(p<0.001)$. AICAR administration significantly decreased $(\sim 1.8$-fold reduction; $p<0.001)$ the number of polyneuronally innervated NMJs found in the IC muscles of SMA animals (Fig. 4F-J"). Moreover, we analyzed the architecture of the NMJ postsynaptic site labeled with $\alpha$-Bgtx and found that, in relation to saline-treated WT mice, IC muscles of SMN $\Delta 7$ mice injected with vehicle displayed a dramatic increase in the number of NMJs showing a plaque morphology and a significant reduction in the number of NMJs exhibiting folds or a perforate appearance (Fig. 4K, L). These structural alterations, reflecting NMJ immaturity in diseased animals, were partially prevented by AICAR treatment [SMN $\Delta 7$-AICAR $v s$ SMN $\Delta 7$-saline $=$ plaque: $\sim 1$.4-fold decrease $(p<0.001)$; folds: $\sim 1.5$-fold increase $(p<0.001)$, perforated: $\sim 5.5$-fold increase $(p<0.001), n=75$ NMJs from $3-4$ animals per experimental condition].

Additionally, we examined whether AICAR was able to modify some presynaptic histopathological changes found in NMJs of SMA mice, such as NF accumulation in nerve terminals and axonal sprouting $[13,61,76]$. AICAR treatment was not able to prevent the abnormal NF aggregation observed in the IC synapses of saline-treated SMN $\Delta 7$ mice (Fig. 4M, O). However, AICAR significantly decreased the number of terminal sprouts found in the IC NMJs of SMN $\Delta 7$ mice ( $\sim 1.2$-fold reduction in the sprouting levels in AICARtreated SMN $\Delta 7$ mice in comparison with those in salineinjected SMN $\Delta 7$ animals; $p<0.001$ ) (Fig. 4N, P).

The neuropeptide CGRP is stored in large dense core vesicles in spinal cord MN somata and nerve terminals, where it appears to have an important role in the development and maintenance of NMJs [77-80]. In agreement with our previous reports [13, 81], CGRP was markedly reduced in spinal cord MNs, and TA and IC NMJs of saline-treated SMN $\Delta 7$ mice, especially at end stages of disease (not shown). This decrease in CGRP content was not significantly modified by AICAR in any tissue samples, as shown in Fig. 4Q-U' for the IC muscle.

\section{AICAR Treatment did not Protect Against MN Death and Reactive Gliosis but Prevented MN Deafferentation in the Spinal Cord of SMN $\Delta 7$ Mice}

Progressive MN loss has been considered a pathological hallmark of SMA. In agreement with our previous report [57], we found a moderate (but significant) MN number depletion ( $\sim 30 \%$ of WT-saline) and MN size reduction $(\sim 15 \%$ of WT-saline) in the spinal cord of saline-treated SMN $\Delta 7$ mice at P8, a time point at which diseased animals exhibited a dramatic impairment in their motor abilities. We then examined whether changes in the skeletal muscle phenotype of SMN $\Delta 7$ mice induced by chronic AICAR administration were accompanied by an increase in MN survival or size. In relation to saline-treated $\operatorname{SMN} \Delta 7$ mice, diseased animals injected with AICAR did not exhibit any changes in the number or size of apparently healthy spinal MNs (Fig. 5A-F).

Although AICAR was not able to protect against MN degeneration in SMA, we wanted to further explore whether AICAR had any beneficial effect on other known histopathological changes in the spinal cord of SMA mice, such as microglial and astroglial reaction in the ventral horn, and the loss of glutamatergic excitatory inputs to MNs $[13,57,74,82$, 83]. Immunocytochemistry with antibodies against IBA1 and GFAP, to stain microglia and astroglia, respectively, revealed that AICAR administration did not prevent the reactive microgliosis and astrogliosis observed in the spinal cord of SMN $\Delta 7$ animals (Fig. 5G-K and L-P, respectively). However, compared with diseased mice injected with saline, AICARtreated SMN $\Delta 7$ animals showed a significant increase $(\sim 1.6$ fold; $p<0.001)$ in the number of VGluT1-positive synapses contacting MN somata (Fig. 5Q-U).

\section{AICAR Treatment did not Modify the NR2A Subunit in Spinal Cord MNs of SMN $\Delta 7$ Mice}

It has been reported that in the type II SMA mouse model, physical exercise is able to increase the expression of the gene encoding the NR2A subunit of the glutamate NMDA receptor in MNs [39]. To examine whether chronic AICAR treatment is able to modulate the expression of NR2A protein in the spinal cord and, particularly in MNs of SMN $\Delta 7$ mice, immuncytochemical and Western blot analyses were performed. In contrast to results by Biondi et al. [39], we did not find a reduction in NR2A expression in lumbar MNs of our SMA mouse model: the quantification of NR2A immunofluorescence in spinal cord sections of WT and $\operatorname{SMN} \Delta 7$ mice treated with saline revealed comparable levels of intensity in both conditions. Similar results were obtained when NR2A protein content was examined in spinal cord extracts by Western blot. Levels of NR2A expression were not significantly modified after AICAR administration (Fig. 6A-O). 

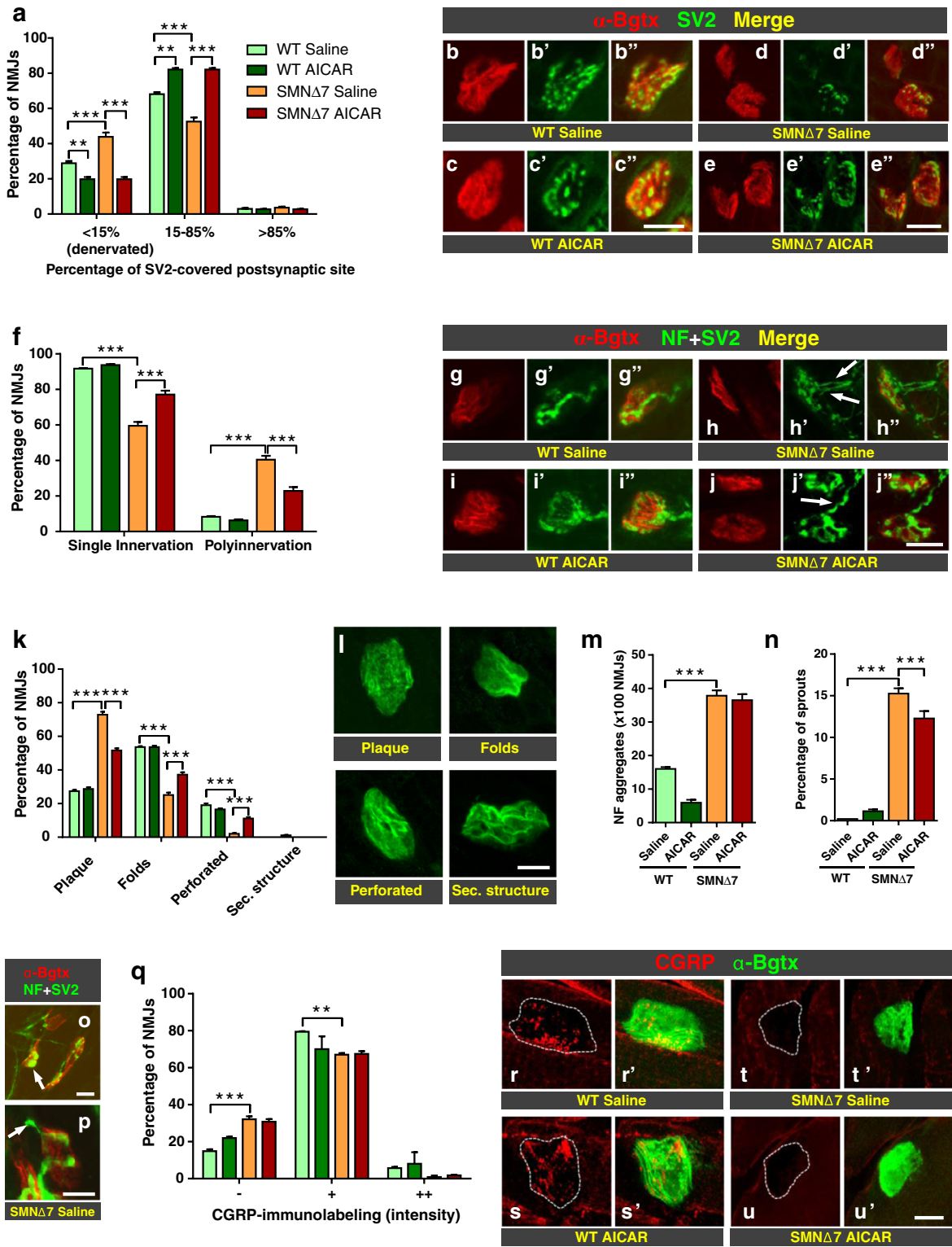

Fig. 4 Effects of chronic 5-aminoimidazole-4-carboxamide-1- $\beta$-Dribofuranoside (AICAR) treatment in the neuromuscular junctions (NMJs) of $S m n^{-/} ; S M N 2^{+/+} ; S M N \Delta 7^{+/+}(\mathrm{SMN} \Delta 7)$ mice. Different presynaptic and postsynaptic structural features were analyzed in NMJs of intercostalis (IC) muscles of postnatal day (P) 8 wildtype (WT) and SMN $\Delta 7$ mice after treatment with either saline or AICAR. (A) Percentage of NMJs showing different degrees of innervation based on the proportion of $\alpha$-bungarotoxin $(\alpha$-Bgtx)labeled postsynaptic site area covered by synaptic vesicle protein 2 (SV2)-immunostained presynaptic terminals. (B-E") Representative images illustrating the results shown in (A); micrographs were taken from sections immunolabeled with SV2 (green) and stained with $\alpha$-Bgtx (red) for postsynaptic site identification. (F) Percentage of NMJs showing single- and multiple innervation. (G-J') Representative images illustrating the results shown in $(\mathrm{F})$; muscle sections were doubleimmunolabeled with SV2 and neurofilament (NF; green) and stained with $\alpha$-Bgtx (red). Arrows in (H') and (J') indicate NMJs (H') double and single innervated in IC muscles of SMN $\Delta 7$ mice treated with (H') saline or (J') AICAR, respectively. (K, L) Percentage of NMJs showing different

degrees of maturity (K) assessed by the morphology of postsynaptic site in form of a homogeneous plaque or containing folds, perforations or secondary structure, as depicted in micrograph images shown in (L). (M, N) Number of NMJs exhibiting (M) NF aggregates and (L) terminal nerve sprouts. $(\mathrm{O}, \mathrm{P})$ Micrographs depicting representative (O, arrow) $\mathrm{NF}$ aggregates and (P, arrow) nerve sprouts in NMJs of IC muscles of SMN $\Delta 7$ mice that were analyzed in the different experimental conditions. (Q) Percentage of NMJs showing different intensities of calcitonin gene-related peptide (CGRP) inmunolabeling. (R-U') Representative images showing CGRP-inmunofluorescence [red in $(\mathrm{R}),(\mathrm{S}),(\mathrm{T})$, and $(\mathrm{U})]$ in NMJs of different experimental conditions; dashed lines delineated NMJs labeled with $\alpha$-Bgtx [green in $\left(\mathrm{R}^{\prime}\right)$, ( $\left.\mathrm{S}^{\prime}\right),\left(\mathrm{T}^{\prime}\right)$, and $\left(\mathrm{U}^{\prime}\right)$ ]. In all graphs, values are shown as mean $\pm \mathrm{SEM} ; * p<0.05, * * p<0.01, * * * p<0.001, n=75$ 100 NMJs of IC muscles from $4-5$ mice per experimental condition (one-way analysis of variance, Bonferroni's post-hoc test); legends corresponding to different experiment groups shown in graphs are indicated in (A). Scale bars: $10 \mu \mathrm{m}$ in (C") (applies to B-C"), (E") (applies to D-E"), (J") (applies to G-J'), (L), (O), (P), and (U') (applies to R-U') 

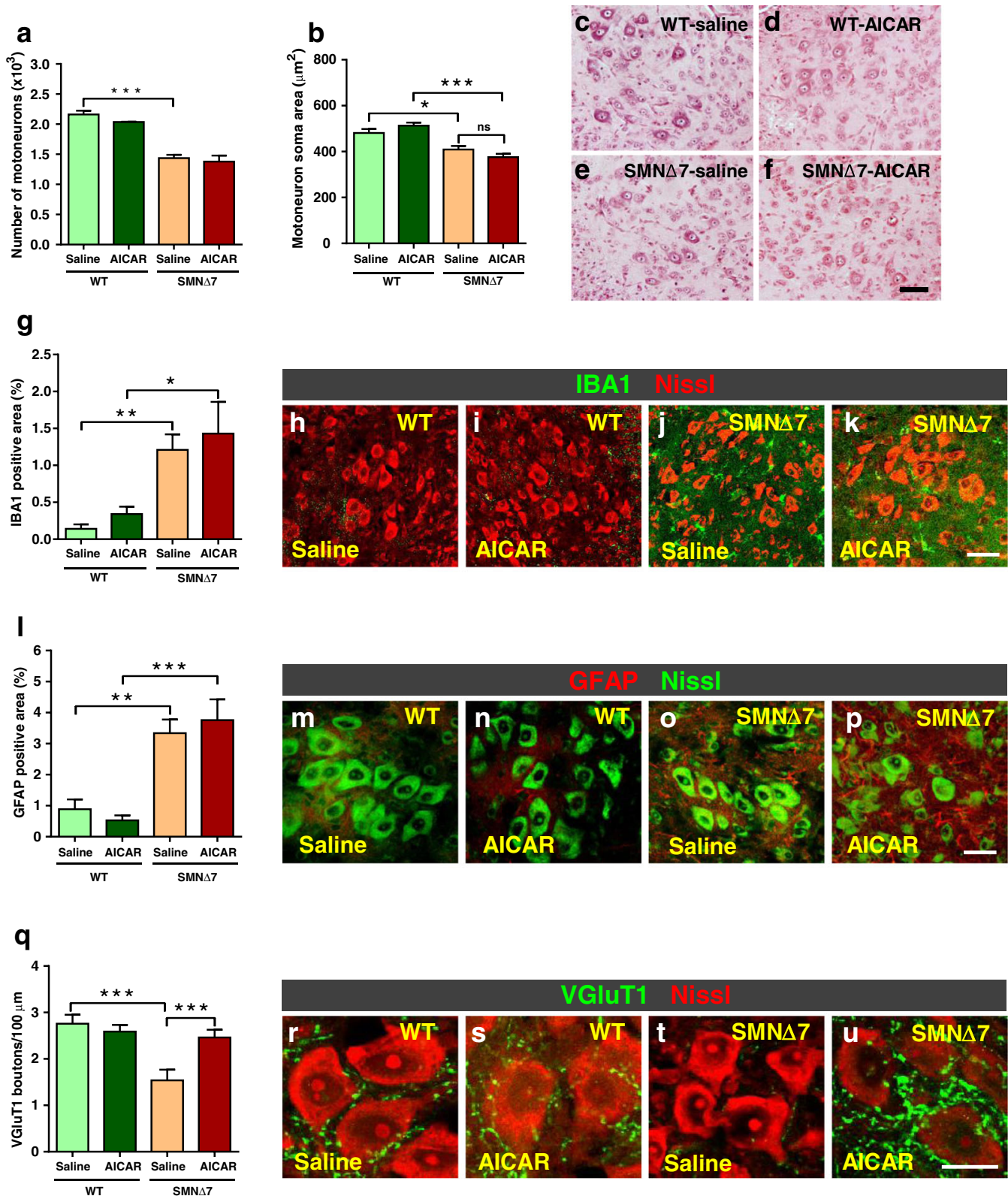

Fig. 5 Chronic treatment with 5-aminoimidazole-4-carboxamide-1$\beta$-D-ribofuranoside (AICAR) does not ameliorate motor neuron (MN) degeneration or the microglial and astroglial reaction observed in the spinal cord of $\mathrm{Smn}^{-/-} ; S M N 2^{+/+} ; S M N \Delta 7^{+/+}$ $(\mathrm{SMN} \Delta 7)$ mice but prevents the loss of excitatory glutamatergic synaptic inputs linked to spinal muscular atrophy (SMA). (A, B) Number of (A) apparently healthy MNs and (B) area of MN soma in postnatal day (P) 8 wild-type (WT) and SMN $\Delta 7$ animals treated with either saline or AICAR. MNs were counted in 5-6 animals per condition; soma area was measured in $120-125 \mathrm{MNs}$ per experimental group. (C-F) Representative micrographs of ventral horn $\mathrm{MNs}$ of $(\mathrm{C}, \mathrm{D}) \mathrm{P} 8 \mathrm{WT}$ and $(\mathrm{E}, \mathrm{F}) \mathrm{SMN} \Delta 7$ mice treated with $(\mathrm{C}, \mathrm{E})$ saline or $(\mathrm{D}, \mathrm{F})$ AICAR; spinal cord paraffin sections were stained with hematoxylin and eosin. (G-P) AICAR treatment does not ameliorate microgliosis or astrogliosis due to SMA. Graphs in $(G)$ and (L) show the quantification of (L) ionized calcium-binding adaptor molecule 1 (IBA1)-positive microglial profiles and glial fibrillary acidic protein (GFAP)positive astroglia around $\mathrm{MNs}$ in the ventral horn of lumbar spinal cord of P8 WT and SMN $\Delta 7$ mice treated with saline or
AICAR; image analysis was performed in 30-35 spinal cord sections per experimental condition (4-6 animals per each genotype and treatment). Representative images of $(\mathrm{H}-\mathrm{K}$, green) IBA1- and (M-P, red) GFAP-immunolabeled profiles around MNs in the spinal cord of mice used for quantification are shown; fluorescent Nissl staining [red in $(\mathrm{H}-\mathrm{K})$, and green in $(\mathrm{M}-\mathrm{P})$ ] was performed to visualize MNs. (Q) Number of vesicular glutamate transporter 1 (VGluT1)-positive boutons apposed to WT and SMN $\Delta 7 \mathrm{MNs}$ per $100 \mu \mathrm{m}$ of soma perimeter in the spinal cord of P8 mice after either saline or AICAR injections; counts were performed in 35-45 MNs per mouse (3-4 mice per experimental condition). (R-U) Confocal images of P8 MNs showing glutamatergic inputs labeled with anti-VGluT1 antibody (green); MNs were visualized with fluorescent Nissl stain (red). Values in graphs represent the mean \pm SEM. ${ }^{*} p<0.05,{ }^{* *} p<0.01$, $* * * p<0.001$ (one-way analysis of variance, Bonferroni's post-hoc test). Scale bars: $50 \mu \mathrm{m}$ in (F) (applies to $\mathrm{C}-\mathrm{F}$ ), $60 \mu \mathrm{m}$ in $(\mathrm{K})$ (applies to $\mathrm{H}-\mathrm{K}$ ); $40 \mu \mathrm{m}$ in (P) (applies to $\mathrm{M}-\mathrm{P}$ ), and $20 \mu \mathrm{m}$ in (U) (applies to R-U) 

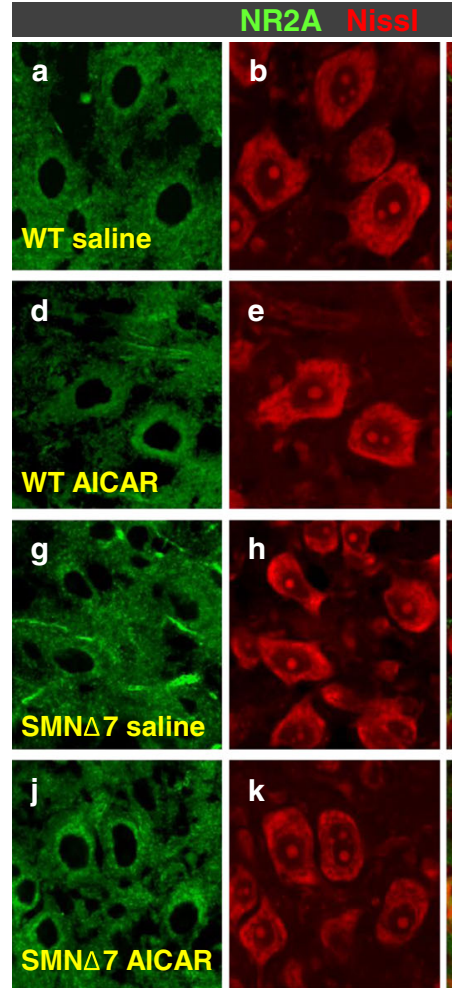
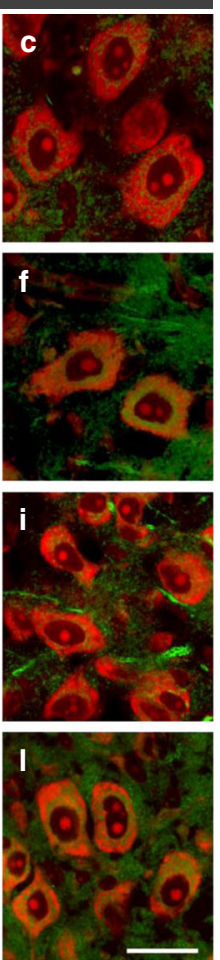

m

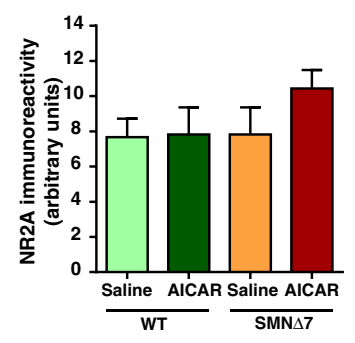

n
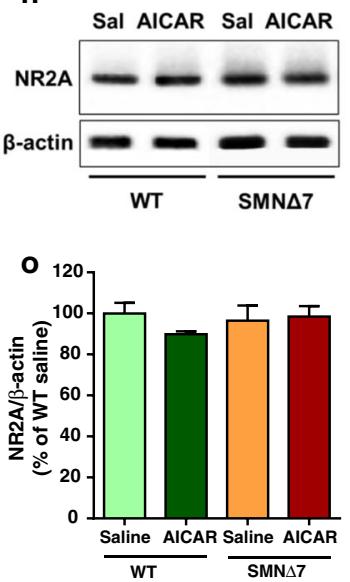

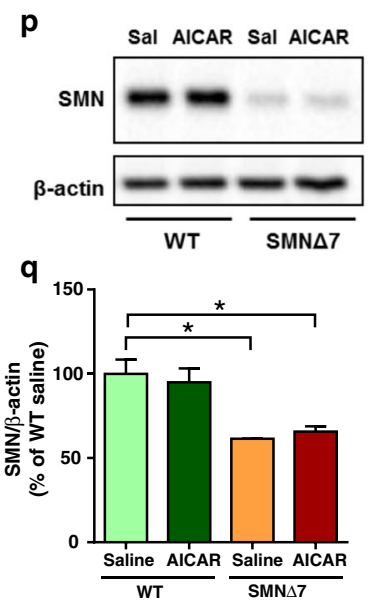

Fig. 6 (A-O) Expression of the $N$-methyl-D-aspartate (NMDA) receptor 2A subunit (NR2A) in lumbar spinal cord of postnatal day (P) 8 wild-type (WT) and $S m n^{-/-} ; S M N 2^{+/+} ; S M N \Delta 7^{+/+}$ $(\mathrm{SMN} \Delta 7)$ mice chronically treated with either saline or 5aminoimidazole-4-carboxamide-1- $\beta$-D-ribofuranoside (AICAR). (A-L) Representative confocal micrographs of spinal cord cryostat sections from (A-C, G-I) saline- and (D-F, J-L) AICAR-treated (A-F) WT and (G-L) SMN $\Delta 7$ mice showing NR2A-immunoreactivity (green) in motor neurons (MNs), visualized with fluorescent Nissl staining (red). (M) Graph showing the quantification NR2A intensity in MN somata of mice subjected to the treatments; $75-85 \mathrm{MNs}$ from 3-4 mice per experimental condition were analyzed. (N) Representative Western blots to determine NR2A expression in spinal cord extracts of mice after different treatments; $\beta$-actin was used as loading control. (O) Densitometry analysis of NR2A protein levels in Western blots; data were obtained from 3-4 mice per

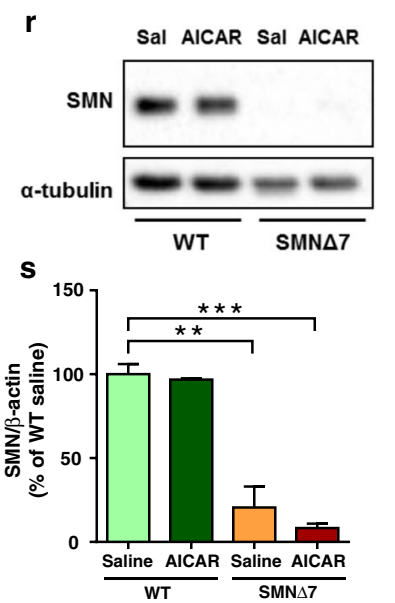

experimental condition and are expressed as the percentage of change in relation to the ratio of NR2A to $\beta$-actin of WT mice treated with saline. (P-S) Western blot assays, performed on $(\mathrm{P})$ lumbar spinal cord and (R) skeletal muscle extracts of P8 WT and $\mathrm{SMN} \Delta 7$ mice following saline or AICAR treatment, demonstrating no changes in survival of motor neuron (SMN) levels induced by the adenosine monophosphate-activated protein kinase agonist. $\beta$-actin and $\alpha$-tubulin were used as loading controls; each sample corresponds to the spinal cord or muscle extract of one animal. (Q, S) Densitometry analysis of SMN protein levels in Western blots; data were obtained from 3 mice per experimental condition and are expressed as the percentage of change in relation to the ratio of SMN to $\beta$-actin or $\alpha$-tubulin of saline-treated WT mice. Values in graphs are shown as mean \pm SEM and were analyzed by using one-way analysis of variance (Bonferroni's post-hoc test). Scale bar in $(\mathrm{L})=30 \mu \mathrm{m}$ (applies to A-L) 


\section{Treatment with AICAR did not Increase the Expression Level of SMN in the Spinal Cord and Skeletal Muscles of SMN $\Delta 7$ Mice}

It has been shown that physical exercise exerts its beneficial effects on SMA mice by increasing the amount of exon 7containing SMN transcripts and, subsequently, the levels of SMN protein in the spinal cord [38]. For this reason, we wanted to determine whether chronic AICAR treatment was able to elevate the expression levels of SMN in the spinal cord and skeletal muscle of SMN $\Delta 7$ mice. Western blot analysis of spinal cord and muscle extracts obtained from AICAR-treated SMN $\Delta 7$ animals revealed similar low levels of SMN found in diseased animals treated with saline (Fig. 6P-S). Therefore, AICAR does not elicit an increase in SMN protein in SMA.

\section{Discussion}

Various studies performed in humans and mouse models suggest that exercise is potentially beneficial in SMA by improving or stabilizing muscle strength and, consequently, motor function $[38,84-86]$. In the present study, we examined the effectiveness of the synthetic AMPK agonist AICAR, an exercise mimetic pharmacological compound [50], as a putative therapeutic agent for SMA in a severe model of the disease, the SMN $\Delta 7$ mouse. We show here that chronic administration of AICAR, starting on the first day after birth, is capable of ameliorating skeletal muscle atrophy and some structural changes found in NMJs of SMA mice. AICAR is also able to prevent the loss of glutamatergic excitatory synaptic afferents on MNs, but did not prevent MN loss or the microglial and astroglial reaction occurring in the spinal cord in the course of disease. Moreover, AICAR did not improve motor behavior or lifespan of SMN $\Delta 7$ mice.

In normal conditions, exercise induces important adaptive changes in the metabolism and gene expression programs of skeletal muscle leading to modifications in its fiber type composition (i.e., type II to type I fiber switch) [71, 87]. AMPK activation appears to play an important role in these muscular changes induced by exercise [45]. AICAR has been reported to be a potent stimulator of AMPK activity in the skeletal muscle [46], and its chronic administration elicits important phenotypic changes in myofibers, such as the shift from a fast (glycolytic) to slow (oxidative) program, which improve exercise performance as demonstrated in distinct animal models (see $[44,88]$ for reviews). In this respect, AICAR has been shown to increase endurance in the absence of physical exercise [50]. In the present study we noticed only a modest capacity of AICAR to stimulate AMPK activation (evaluated by kinase phosphorylation) in the skeletal muscle of WT mice in vivo. This is in contrast to the strong effect of AICAR promoting AMPK activity in $\mathrm{C}_{2} \mathrm{C}_{12}$ muscle cells found in vitro in a previous study [51]. Although these authors did not examine the activation of AMPK in vivo, they reported beneficial effects of AICAR in skeletal muscles of the $m d x$ mouse (a model of Duchenne muscular dystrophy) when chronically administered at the same dose, regimen, and mode of injection as used here. Indeed, we found phenotypic changes in the skeletal muscle and NMJ (as discussed below) of SMN $\Delta 7$ mice after AICAR administration, suggesting a pharmacological effect of the agent in our model, despite moderate AMPK activation. In fact, it is known that AICAR is not a selective activator of AMPK and can also stimulate other AMPsensitive enzymes, which play an important role in the regulation of muscle metabolism [89]. Interestingly, we found that muscles from SMN $\Delta 7$ mice have a basal increase in AMPK activity that is not significantly modified by AICAR treatment, similar to what has been reported in dystrophic muscles from $m d x$ mice [62]. Muscle dysfunction, linked to SMA, could account for the elevated AMPK activity found in our model. According to this possibility, increases in the expression and activity of different kinases have been observed in a variety of muscle pathologies [62, 90-92].

We found that AICAR ameliorates muscular atrophy in SMN $\Delta 7$ as a consequence of the increase in myofiber size, which appears to be responsible for the modest increase in body weight of diseased animals treated with the compound. This is in contrast to the absence of changes in the crosssectional area of myofibers reported in the dystrophic muscle of $m d x$ mice following chronic treatment with AICAR [51]. Differences in the physiological and histopathological alterations occurring in muscles affected by SMA and in dystrophic muscles (see, e.g., [93]) could account for the discrepancies existing between these mouse models regarding the effects of AICAR on myofiber size. However, in agreement with findings reported in $m d x$ mice [51], we also observed that AICAR increases the proportion of type I slow-twitch myofibers in SMN $\Delta 7$ mice. It is important to note that in basal (saline-treated) conditions we observed that muscles from $\mathrm{SMN} \Delta 7$ mice had a dramatic increase in the proportion of type I fibers in a predominantly fast muscle, the TA. This change could be the consequence of a metabolic oxidative myogenic compensatory program intended to limit muscle dysfunction resulting from the disease. Similar changes in the myofiber typology have been reported in hindlimb muscles other than TA [69]. Type I (slow, oxidative) myofibers, which have slow isoforms of contractile proteins, are more resistant to fatigue than type II (fast, glycolytic) myofibers, and skeletal muscles with a higher content of type I fibers are more resistant to wasting than those with a predominance of type II fibers [64, 94]. As AICAR promotes an oxidative response in skeletal muscle [44], the increase in the proportion of type I fibers in SMA may be beneficial by favoring the oxidative phenotype of myofibers that mitigate the consequences of muscular denervation. 
It has been documented that regular physical exercise has a beneficial effect in a mouse model of SMA type II $\left(\mathrm{Smn}^{-1-} ; \mathrm{SMN} 2[2 \mathrm{Hung}]^{+/+}\right.$mice) by increasing the lifespan, reducing MN degeneration, and limiting the muscular atrophy characteristic of the disease. This therapeutic action has been attributed to the elevation of SMN levels, which appears to be the result of an increase in exon 7containing SMN transcripts, as has been reported in the spinal cord of SMA-trained mice [38]. In our study, we found that AICAR did not elicit any beneficial effects on $\mathrm{MN}$ degeneration or reactive gliosis occurring in spinal cord of SMN $\Delta 7$ mice [57], indicating that AICAR is not as effective as physical exercise, in promoting MN survival in SMA affected animals. The absence of changes in SMN protein levels we observed in the spinal cord and skeletal muscle of SMN $\Delta 7$ mice after AICAR administration may account for these differences. It is known that the expression of SMN at prenatal and early postnatal stages is crucial for MN survival and the normal development of motor units, as well as for the refinement and maturation of NMJs [95-97]. Although AICAR was not able to promote MN survival in SMA animals, this compound did ameliorate some NMJ alterations linked to the disease. Thus, we observed that AICAR mitigated denervation and favored the maturation of SMA NMJs, as determined by the reduction in polyneuronal innervation and the achievement of a more mature architecture (i.e., pretzel-like morphology) of postsynaptic sites. Moreover, AICAR reduced axonal sprouting observed in NMJs of SMN $\Delta 7$ mice. As no elevation in muscle SMN protein levels was found in AICAR-treated SMN $\Delta 7$ animals, these beneficial effects on NMJs, which occur in a SMN-independent manner, appear to be a consequence of a direct action of AICAR on skeletal muscle. In fact, it has been shown that AICAR alters the content of different muscle phenotypic modifiers (including the transcriptional coactivator PGC- $1 \alpha$, the nuclear receptor peroxisome proliferator-activated receptor $\delta$, the nicotinamide adenine dinucleotide-dependent protein deacetylase sirtuin1 , and the receptor-interacting protein 140), which appear to be, at least in part, responsible for AICAR functional improvements on dystrophic muscle [44, 51]. PGC- $1 \alpha$, either directly or through the activation of the neuregulin-1-dependent pathway, induces the transcription of synaptic genes that play a key role in the formation of acetylcholine receptor clusters and NMJ maintenance [98]. PGC- $1 \alpha$ expression has been shown to be increased in muscle after physical exercise $([66,99]$, see also [100] for a review), and in normal and dystrophic muscles of mice chronically treated with AICAR $[50,51]$. The latter results contrast with the absence of a substantial increase in PGC- $1 \alpha$ protein content induced by AICAR in our study. The age of the animals and the time frame of
AICAR administration could explain the disparity of results; thus, adult animals and longer periods of treatment ( $\sim 30$ days) have been used in the previous studies [50, 51]. In fact, we noticed a moderate increase in PGC- $1 \alpha$ band density in muscles from some mice (both WT and $\mathrm{SMN} \Delta 7$ ) treated with the compound, although, owing to sample variability, no statistically significant changes were observed when individual data were pooled. However, in agreement with our findings, no changes in PGC- $1 \alpha$ mRNA expression have been reported in $m d x$ mice subjected to chronic AICAR treatment. Whether the activation of phenotypic modifiers other than PGC- $1 \alpha$ or additional mechanisms, such as the expression of neurotrophic factors, are important as mediators of the AICAR actions we describe here, needs further investigation. In this respect, it is well known that exercise induces the expression of several neurotrophic factors including insulin-like growth factor-1, neurotrophin-4, brain-derived neurotrophic factor, and glial cell line-derived neurotrophic factor that are important in maintaining the integrity of NMJs [100].

MN loss has been considered a histopathological hallmark of human SMA, which has also been found to occur in mouse models of this disease. However, in contrast to the extensive spinal MN loss reported in humans [2], most of the studies performed on SMA mice, including the SMN $\Delta 7$ model, have shown that, even at end stages of disease, $\mathrm{MN}$ death is modest and does not account for the degree of muscular weakness and paralysis exhibited by these animals [13, 55, 57, 61, 81]. Nevertheless, surviving MNs in SMA mice display alterations indicative of cellular dysfunction, which include the loss of glutamatergic (VGluT1 immunoreactive) synaptic afferents $[57,74,81-83]$. We and others have reported that this glutamatergic deafferentation precedes $\mathrm{MN}$ death and begins at prenatal stages in $\mathrm{SMN} \Delta 7$ animals $[57,83]$, long before $\mathrm{MN}$ loss is observed. However, whether or not MN deafferentation modifies cell death in SMA is not clear, and may, in fact, be a consequence of primary MN pathology [61, 101, 102]. In this regard, it has been recently reported that SMN deficiency results in intrinsic changes in the electrophysiological properties of MNs [103], and that replacement of SMN only in MNs is necessary and sufficient to restore the functional deficits of motor units [104]. These findings suggest that adequate levels of SMN in MNs are crucial to avoid the dysfunction and subsequent degeneration of MNs. Here we show that the loss of VGluT1 synapses on MNs of SMN $\Delta 7$ mice can be prevented by chronic AICAR administration. Nevertheless, this improvement in central synaptic connectivity is not sufficient to prevent the progressive MN loss that takes place in the course of the disease. As VGluT1 is expressed in synaptic boutons on MNs mainly derived from proprioceptive sensory axons [105], it is possible that, at least in part, the effect of AICAR in preventing the glutamatergic deafferentation is due to the action of this compound on 
skeletal muscle and, particularly, on muscle spindles. In fact, it has been shown that there is a loss of proprioceptive, muscle spindle inputs to MNs, which occurs early in SMA and appears to be secondary to $\mathrm{MN}$ dysfunction $[83,102]$. Whether AICAR is able to modify muscle spindles in SMN $\Delta 7$ requires further detailed studies.

It is important to note that SMA is a complex and multisystemic disorder, although it is phenotypically expressed mainly as a neuromuscular disease [13, 61, 106, 107]. It appears that muscle and nerve may be independently affected by SMN deficiency [17, 107], and muscle has only a minor impact on SMA pathogenesis. Indeed, several studies have reported that improvements in skeletal muscle and NMJ morphology and physiology, after SMN replacement in MNs, do not result in a significant increase in survival $[61,82,101$, $104,108,109]$. Moreover, it has been recently shown that low levels of SMN in muscle are sufficient for its normal function, and muscular replacement of SMN does not modify the lifespan of SMA [110]. Nevertheless, these results should be interpreted cautiously as a conspicuous myopathy has been reported to occur after deletion of murine $S M N$ exon 7 in the skeletal muscle [11]. In any case, an intricate crosstalk between the $\mathrm{MN}$ and muscle occurs in normal and pathological conditions. As our results suggest that AICAR mainly targets the muscular side of the system, it would be interesting to examine whether the association of this compound with $\mathrm{MN}$-directed therapies results in a more remarkable benefit in SMA clinical outcome. Perhaps, an AICAR assay in a less severe model of this disease, such as the $S m n^{2 B /-}$ mouse [76, 111], may further clarify the benefits of this compound as a muscle-directed therapy in the context of SMA.

Acknowledgments We thank Dr. Ronald W. Oppenheim for critical reading of the manuscript and for helpful comments and suggestions; Dr. Anna Casanovas and Dr. Sara Hernández for useful discussions; and Marta Hereu and Alexandra Eritja for technical assistance. We also thank Javier Sábado, Alba Blasco, and Ariadna Salvador for their help with some experiments of this study. This work was supported by grants from the Ministerio de Economía y Competitividad cofinanced by FEDER (SAF2012-31831 to J.C., and SAF2011-22908 to J.E.E.).

\section{References}

1. Emery AE. Population frequencies of inherited neuromuscular diseases - a world survey. Neuromuscul Disord 1991;1:19-29.

2. Crawford TO, Pardo CA. The neurobiology of childhood spinal muscular atrophy. Neurobiol Dis 1996;3:97-110.

3. Lefebvre S, Burglen L, Reboullet S, et al. Identification and characterization of a spinal muscular atrophy-determining gene. Cell 1995;80:155-165.

4. Pellizzoni L, Kataoka N, Charroux B, Dreyfuss G. A novel function for SMN, the spinal muscular atrophy disease gene product, in pre-mRNA splicing. Cell 1998;95:615-624.

5. Kolb SJ, Battle DJ, Dreyfuss G. Molecular functions of the SMN complex. J Child Neurol 2007;22:990-994.
6. Lorson CL, Hahnen E, Androphy EJ, Wirth B. A single nucleotide in the SMN gene regulates splicing and is responsible for spinal muscular atrophy. Proc Natl Acad Sci U S A 1999;96:6307-6011.

7. Lefebvre S, Burlet P, Liu Q, et al. Correlation between severity and SMN protein level in spinal muscular atrophy. Nat Genet 1997;16: 265-269.

8. Coovert DD, Le TT, McAndrew PE, et al. The survival motor neuron protein in spinal muscular atrophy. Hum Mol Gen 1997;6:1205-1214.

9. Burghes AH, Beattie CE. Spinal muscular atrophy: why do low levels of survival motor neuron protein make motor neurons sick? Nat Rev Neurosci 2009;10:597-609.

10. Dombert B, Sivadasan R, Simon CM, Jablonka S, Sendtner M. Presynaptic localization of Smn and hnRNP R in axon terminals of embryonic and postnatal mouse motoneurons. PLoS One 2014;9:e110846.

11. Cifuentes-Diaz C, Frugier T, Tiziano FD, et al. Deletion of murine SMN exon 7 directed to skeletal muscle leads to severe muscular dystrophy. J Cell Biol 2001;152:1107-1114.

12. Arnold AS, Gueye M, Guettier-Sigrist S, et al. Reduced expression of nicotinic AChRs in myotubes from spinal muscular atrophy I patients. Lab Invest 2004;84:1271-1278.

13. Dachs E, Hereu M, Piedrafita L, Casanovas A, Calderó J, Esquerda JE. Defective neuromuscular junction organization and postnatal myogenesis in mice with severe spinal muscular atrophy. J Neuropathol Exp Neurol 2011;70:444-461.

14. Boyer JG, Deguise MO, Murray LM, et al. Myogenic program dysregulation is contributory to disease pathogenesis in spinal muscular atrophy. Hum Mol Gen 2014;23:4249-4259.

15. Bricceno KV, Martinez T, Leikina E, et al. Survival motor neuron protein deficiency impairs myotube formation by altering myogenic gene expression and focal adhesion dynamics. Hum Mol Gen 2014;23:4745-4757.

16. Fayzullina S, Martin LJ. Skeletal muscle DNA damage precedes spinal motor neuron DNA damage in a mouse model of Spinal Muscular Atrophy (SMA). PLoS One 2014;9:e93329.

17. Boyer JG, Ferrier A, Kothary R. More than a bystander: the contributions of intrinsic skeletal muscle defects in motor neuron diseases. Front Physiol 2013;4:356.

18. Pruss RM, Giraudon-Paoli M, Morozova S, Berna P, Abitbol JL, Bordet T. Drug discovery and development for spinal muscular atrophy: lessons from screening approaches and future challenges for clinical development. Future Med Chem 2010;2:1429-1440.

19. Tsai LK. Therapy development for spinal muscular atrophy in SMN independent targets. Neural Plast 2012;2012:456478.

20. Seo J, Howell MD, Singh NN, Singh RN. Spinal muscular atrophy: an update on therapeutic progress. Biochim Biophys Acta 2013; 1832:2180-2190.

21. d'Ydewalle C, Sumner CJ. Spinal muscular atrophy therapeutics: where do we stand? Neurotherapeutics 2015;12:303-316.

22. Makhortova NR, Hayhurst M, Cerqueira A, et al. A screen for regulators of survival of motor neuron protein levels. Nat Chem Biol 2011;7:544-552.

23. Chen PC, Gaisina IN, El-Khodor BF, et al. Identification of a maleimide-mased glycogen synthase kinase-3 (GSK-3) inhibitor, BIP-135, that prolongs the median survival time of delta7 SMA KO mouse model of spinal muscular atrophy. ACS Chem Neurosci 2012;3:5-11.

24. Markowitz JA, Singh P, Darras BT. Spinal muscular atrophy: a clinical and research update. Pediatr Neurol 2012;46:1-12.

25. Cherry JJ, Kobayashi DT, Lynes MM, et al. Assays for the identification and prioritization of drug candidates for spinal muscular atrophy. Assay Drug Dev Technol 2014;12:315-341.

26. Iascone DM, Henderson CE, Lee JC. Spinal muscular atrophy: from tissue specificity to therapeutic strategies. F1000Prime Rep 2015;7:04. 
27. Monani UR, De Vivo DC. Neurodegeneration in spinal muscular atrophy: from disease phenotype and animal models to therapeutic strategies and beyond. Future Neurol 2014;9:49-65.

28. Burghes AH, McGovern VL. Antisense oligonucleotides and spinal muscular atrophy: skipping along. Genes Dev 2010;24:15741579.

29. Passini MA, Bu J, Richards AM, et al. Antisense oligonucleotides delivered to the mouse CNS ameliorate symptoms of severe spinal muscular atrophy. Sci Transl Med 2011;3:72ra18.

30. Rigo F, Hua Y, Krainer AR, Bennett CF. Antisense-based therapy for the treatment of spinal muscular atrophy. J Cell Biol 2012;199: 21-25.

31. Douglas AG, Wood MJ. Splicing therapy for neuromuscular disease. Mol Cell Neurosci 2013;56:169-185.

32. Sivanesan S, Howell MD, Didonato CJ, Singh RN. Antisense oligonucleotide mediated therapy of spinal muscular atrophy. Transl Neurosci 2013;4.

33. Wyatt TJ, Keirstead HS. Stem cell-derived neurotrophic support for the neuromuscular junction in spinal muscular atrophy. Expert Opin Biol Ther 2010;10:1587-1594.

34. Gowing G, Svendsen CN. Stem cell transplantation for motor neuron disease: current approaches and future perspectives. Neurother 2011;8:591-606.

35. Kirkinezos IG, Hernandez D, Bradley WG, Moraes CT. Regular exercise is beneficial to a mouse model of amyotrophic lateral sclerosis. Ann of Neurol 2003;53:804-807.

36. Liebetanz D HK, von Lewinski F, Kahler E, Paulus W. Extensive exercise is not harmful in amyotrophic lateral sclerosis. Eur J Neurosci 2004;20:3115-3120.

37. Mahoney DJ, Rodriguez C, Devries M, Yasuda N, Tarnopolsky MA. Effects of high-intensity endurance exercise training in the G93A mouse model of amyotrophic lateral sclerosis. Muscle Nerve 2004;29:656-662.

38. Grondard C, Biondi O, Armand AS, et al. Regular exercise prolongs survival in a type 2 spinal muscular atrophy model mouse. J Neurosci 2005;25:7615-7622.

39. Biondi O, Grondard C, Lecolle S, et al. Exercise-induced activation of NMDA receptor promotes motor unit development and survival in a type 2 spinal muscular atrophy model mouse. J Neurosci 2008;28:953-962.

40. Andreassi C, Patrizi AL, Monani UR, Burghes AH, Brahe C, Eboli ML. Expression of the survival of motor neuron (SMN) gene in primary neurons and increase in SMN levels by activation of the N-methyl-D-aspartate glutamate receptor. Neurogenetics 2002;4:29-36.

41. Fujii N, Hayashi T, Hirshman MF, et al. Exercise induces isoformspecific increase in 5'AMP-activated protein kinase activity in human skeletal muscle. Biochem Biophys Res Commun 2000;273:1150-1155.

42. Chen ZP, Stephens TJ, Murthy S, et al. Effect of exercise intensity on skeletal muscle AMPK signaling in humans. Diabetes 2003;52: 2205-2212.

43. Mu J, Brozinick JT, Jr., Valladares O, Bucan M, Birnbaum MJ. A role for AMP-activated protein kinase in contraction- and hypoxia-regulated glucose transport in skeletal muscle. Mol Cell 2001;7:1085-1094.

44. Ljubicic V, Burt M, Jasmin BJ. The therapeutic potential of skeletal muscle plasticity in Duchenne muscular dystrophy: phenotypic modifiers as pharmacologic targets. FASEB J 2014;28:548-568.

45. Jorgensen SB, Richter EA, Wojtaszewski JF. Role of AMPK in skeletal muscle metabolic regulation and adaptation in relation to exercise. J Physiol 2006;574:17-31.

46. Merrill GF, Kurth EJ, Hardie DG, Winder WW. AICA riboside increases AMP-activated protein kinase, fatty acid oxidation, and glucose uptake in rat muscle. Am J Physiol 1997;273:E1107E1112.
47. Holmes BF, Kurth-Kraczek EJ, Winder WW. Chronic activation of 5'-AMP-activated protein kinase increases GLUT-4, hexokinase, and glycogen in muscle. J Appl Physiol 1999;87:1990-1995.

48. Winder WW, Holmes BF, Rubink DS, Jensen EB, Chen M, Holloszy JO. Activation of AMP-activated protein kinase increases mitochondrial enzymes in skeletal muscle. J Appl Physiol 2000;88:2219-2226.

49. Yan Z, Okutsu M, Akhtar YN, Lira VA. Regulation of exerciseinduced fiber type transformation, mitochondrial biogenesis, and angiogenesis in skeletal muscle. J Appl Physiol 2011;110:264274.

50. Narkar VA, Downes M, Yu RT, et al. AMPK and PPARdelta agonists are exercise mimetics. Cell 2008;134:405-415.

51. Ljubicic V, Miura P, Burt M, et al. Chronic AMPK activation evokes the slow, oxidative myogenic program and triggers beneficial adaptations in mdx mouse skeletal muscle. Hum Mol Gen 2011;20:3478-3493.

52. Bueno Junior CR, Pantaleao LC, Voltarelli VA, Bozi LH, Brum PC, Zatz M. Combined effect of AMPK/PPAR agonists and exercise training in $\mathrm{mdx}$ mice functional performance. PLoS One 2012;7:e45699.

53. Jahnke VE, Van Der Meulen JH, Johnston HK, et al. Metabolic remodeling agents show beneficial effects in the dystrophindeficient mdx mouse model. Skelet Muscle 2012;2:16.

54. Pauly M, Daussin F, Burelle Y, et al. AMPK activation stimulates autophagy and ameliorates muscular dystrophy in the mdx mouse diaphragm. Am J Pathol 2012;181:583-592.

55. Le TT, Pham LT, Butchbach ME, et al. SMNDelta7, the major product of the centromeric survival motor neuron (SMN2) gene, extends survival in mice with spinal muscular atrophy and associates with full-length SMN. Hum Mol Gen 2005;14:845-857.

56. Sleigh JN, Gillingwater TH, Talbot K. The contribution of mouse models to understanding the pathogenesis of spinal muscular atrophy. Dis Model Mech 2011;4:457-467.

57. Tarabal O, Caraballo-Miralles V, Cardona-Rossinyol A, et al. Mechanisms involved in spinal cord central synapse loss in a mouse model of spinal muscular atrophy. J Neuropathol Exp Neurol 2014;73:519-535.

58. El-Khodor BF, Edgar N, Chen A, et al. Identification of a battery of tests for drug candidate evaluation in the SMNDelta7 neonate model of spinal muscular atrophy. Exp Neurol 2008;212:29-43.

59. Clarke PGH, Oppenheim RW. Neuron death in vertebrate development - in-vivo methods. Methods Cell Biol 1995;46:277-321.

60. Calderó J, Ciutat D, Lladó J, Castán E, Oppenheim RW, Esquerda JE. Effects of excitatory amino acids on neuromuscular development in the chick embryo. J Comp Neurol 1997;387:73-95.

61. Gogliotti RG, Quinlan KA, Barlow CB, Heier CR, Heckman CJ, Didonato CJ. Motor neuron rescue in spinal muscular atrophy mice demonstrates that sensory-motor defects are a consequence, not a cause, of motor neuron dysfunction. J Neurosci 2012;32: 3818-3829.

62. Ljubicic V, Khogali S, Renaud JM, Jasmin BJ. Chronic AMPK stimulation attenuates adaptive signaling in dystrophic skeletal muscle. Am J Physiol Cell Physiol 2012;302:C110-C121.

63. Scarpulla RC. Metabolic control of mitochondrial biogenesis through the PGC-1 family regulatory network. Biochim Biophys Acta 2011;1813:1269-1278.

64. Schiaffino S, Reggiani C. Fiber types in mammalian skeletal muscles. Physiol Rev 2011;91:1447-1531.

65. Baar K, Wende AR, Jones TE, Marison M, Nolte LA, Chen M. Adaptations of skeletal muscle to exercise: rapid increase in the transcriptional coactivator PGC-1. FASEB J 2002; 16:1879-1886.

66. Russell AP, Feilchenfeldt J, Schreiber S, et al. Endurance training in humans leads to fiber type-specific increases in levels of peroxisome proliferator-activated receptor-gamma coactivator-1 and 
peroxisome proliferator-activated receptor-alpha in skeletal muscle. Diabetes 2003;52:2874-2881.

67. Terada S, Goto M, Kato M, Kawanaka K, Shimokawa T, Tabata I. Effects of low-intensity prolonged exercise on PGC-1 mRNA expression in rat epitrochlearis muscle. Biochem Biophys Res Commun 2002;296:350-354.

68. Jager S, Handschin C, St-Pierre J, Spiegelman B. M. AMPactivated protein kinase (AMPK) action in skeletal muscle via direct phosphorylation of PGC-1alpha. Proc Natl Acad Sci U S A 2007;104:12017-12022.

69. Lee YI, Mikesh M, Smith I, Rimer M, Thompson W. Muscles in a mouse model of spinal muscular atrophy show profound defects in neuromuscular development even in the absence of failure in neuromuscular transmission or loss of motor neurons. Dev Biol 2011;356:432-444

70. Kong L, Wang X, Choe DW, et al. Impaired synaptic vesicle release and immaturity of neuromuscular junctions in spinal muscular atrophy mice. J Neurosci 2009;29:842-851.

71. Pette D, Staron RS. Myosin isoforms, muscle fiber types, and transitions. Microsc Res Tech 2000;50:500-509.

72. Hamalainen N, Pette D. The histochemical profiles of fast fiber types IIB, IID, and IIA in skeletal muscles of mouse, rat, and rabbit. J Histochem Cytochem 1993;41:733-743.

73. Ling KK, Gibbs RM, Feng Z, Ko CP. Severe neuromuscular denervation of clinically relevant muscles in a mouse model of spinal muscular atrophy. Hum Mol Gen 2012;21:185-195.

74. Ling KK, Lin MY, Zingg B, Feng Z, Ko CP. Synaptic defects in the spinal and neuromuscular circuitry in a mouse model of spinal muscular atrophy. PLoS One 2010;5:e15457.

75. Murray LM, Comley LH, Thomson D, Parkinson N, Talbot K, Gillingwater TH. Selective vulnerability of motor neurons and dissociation of pre- and post-synaptic pathology at the neuromuscular junction in mouse models of spinal muscular atrophy. Hum Mol Gen 2008; 17:949-962.

76. Murray LM, Beauvais A, Bhanot K, Kothary R. Defects in neuromuscular junction remodelling in the $\operatorname{Smn}(2 \mathrm{~B} /-)$ mouse model of spinal muscular atrophy. Neurobiol Dis 2013;49:57-67.

77. Matteoli M, Balbi S, Sala C, et al. Developmentally regulated expression of calcitonin gene-related peptide at mammalian neuromuscular-junction. J Mol Neurosci 1990;2:175-184.

78. Sala C, Andreose JS, Fumagalli G, Lomo T. Calcitonin generelated peptide: possible role in formation and maintenance of neuromuscular junctions. J Neurosci 1995;15:520-528.

79. Tarabal O, Calderó J, Esquerda JE. Intramuscular nerve sprouting induced by CNTF is associated with increases in CGRP content in mouse motor nerve terminals. Neurosci Lett 1996;219:60-64.

80. Tarabal O, Calderó J, Ribera J, et al. Regulation of motoneuronal calcitonin gene-related peptide (CGRP) during axonal growth and neuromuscular synaptic plasticity induced by botulinum toxin in rats. Eur J Neurosci 1996;8:829-836.

81. Dachs E, Piedrafita L, Hereu M, Esquerda JE, Calderó J. Chronic treatment with lithium does not improve neuromuscular phenotype in a mouse model of severe spinal muscular atrophy. Neuroscience 2013;250:417-433.

82. Park GH, Maeno-Hikichi Y, Awano T, Landmesser LT, Monani UR. Reduced survival of motor neuron (SMN) protein in motor neuronal progenitors functions cell autonomously to cause spinal muscular atrophy in model mice expressing the human centromeric (SMN2) gene. J Neurosci 2010;30:12005-12019.

83. Mentis GZ, Blivis D, Liu W, et al. Early functional impairment of sensory-motor connectivity in a mouse model of spinal muscular atrophy. Neuron 2011;69:453-467.

84. McCartney N, Moroz D, Garner SH, McComas AJ. The effects of strength training in patients with selected neuromuscular disorders. Med Sci Sports Exerc 1988;20:362-368.
85. Lewelt A, Krosschell KJ, Stoddard GJ, et al. Resistance Strength Training Exercise in Children with Spinal Muscular Atrophy. Muscle Nerve 2015;52:559-567.

86. Madsen KL, Hansen RS, Preisler N, Thogersen F, Berthelsen MP, Vissing J. Training improves oxidative capacity, but not function in Spinal Muscular Atrophy Type III. Muscle Nerve 2015;52:240244.

87. Pette D, Staron RS. Mammalian skeletal muscle fiber type transitions. Int Rev Cytol 1997;170:143-223.

88. Ljubicic V, Jasmin BJ. AMP-activated protein kinase at the nexus of therapeutic skeletal muscle plasticity in Duchenne muscular dystrophy. Trends Mol Med 2013;19:614-624.

89. Witczak CA, Sharoff CG, Goodyear LJ. AMP-activated protein kinase in skeletal muscle: From structure and localization to its role as a master regulator of cellular metabolism. Cell Mol Life Sci 2008;65:3737-3755.

90. Mylabathula DB, Rice KM, Wang Z, Uddemarri S, Kinnard RS, Blough ER. Age-associated changes in MAPK activation in fastand slow-twitch skeletal muscle of the F344/NNiaHSD X Brown Norway/BiNia rat model. Exp Gerontol 2006;41:205-214.

91. Singh R, Millman G, Turin E, et al. Increases in nuclear p65 activation in dystrophic skeletal muscle are secondary to increases in the cellular expression of p65 and are not solely produced by increases in IkappaB-alpha kinase activity. J Neurol Sci 2009;285: 159-171.

92. Thomson DM, Brown JD, Fillmore N, et al. AMP-activated protein kinase response to contractions and treatment with the AMPK activator AICAR in young adult and old skeletal muscle. Journal Physiol 2009;587:2077-2086.

93. Dubowitz V, Sewry CA. Muscle biopsy: a practical approach. 3rd ed. Saunders Elsevier, Philadelphia, PA, 2007.

94. Minnaard R, Drost MR, Wagenmakers AJ, van Kranenburg GP, Kuipers H, Hesselink MK. Skeletal muscle wasting and contractile performance in septic rats. Muscle Nerve 2005;31:339-348.

95. Le TT, McGovern VL, Alwine IE, et al. Temporal requirement for high SMN expression in SMA mice. Hum Mol Gen 2011;20: 3578-3591.

96. Lutz CM, Kariya S, Patruni S, et al. Postsymptomatic restoration of SMN rescues the disease phenotype in a mouse model of severe spinal muscular atrophy. J Clin Invest 2011;121:3029-3041.

97. Kariya S, Obis T, Garone C, et al. Requirement of enhanced Survival Motoneuron protein imposed during neuromuscular junction maturation. J Clin Invest 2014;124:785-800.

98. Handschin C, Kobayashi YM, Chin S, Seale P, Campbell KP, Spiegelman BM. PGC-1alpha regulates the neuromuscular junction program and ameliorates Duchenne muscular dystrophy. Genes Dev 2007;21:770-783.

99. Goto M, Terada S, Kato M, et al. cDNA Cloning and mRNA analysis of PGC-1 in epitrochlearis muscle in swimmingexercised rats. Biochem Biophys Res Commun 2000;274:350354.

100. Nishimune H, Stanford JA, Mori Y. Role of exercise in maintaining the integrity of the neuromuscular junction. Muscle Nerve 2014;49:315-324.

101. Martinez TL, Kong L, Wang X, et al. Survival motor neuron protein in motor neurons determines synaptic integrity in spinal muscular atrophy. J Neurosci 2012;32:8703-8715.

102. Thirumalai V, Behrend RM, Birineni S, Liu W, Blivis D, O'Donovan MJ. Preservation of VGLUT1 synapses on ventral calbindinimmunoreactive interneurons and normal locomotor function in a mouse model of spinal muscular atrophy. J Neurophysiol 2013;109: 702-710.

103. Liu H, Lu J, Chen H, Du Z, Li XJ, Zhang S. Spinal muscular atrophy patient-derived motor neurons exhibit hyperexcitability. Sci Rep 2015;5:12189. 
104. McGovern VL, Iyer CC, Arnold WD, et al. SMN expression is required in motor neurons to rescue electrophysiological deficits in the SMNDelta7 mouse model of SMA. Hum Mol Genet 2015;24:5524-5541.

105. Alvarez FJ, Titus-Mitchell HE, Bullinger KL, Kraszpulski M, Nardelli P, Cope TC. Permanent central synaptic disconnection of proprioceptors after nerve injury and regeneration. I. Loss of VGLUT1/IA synapses on motoneurons. J Neurophysiol 2011;106:2450-2470.

106. Schreml J, Riessland M, Paterno M, et al. Severe SMA mice show organ impairment that cannot be rescued by therapy with the HDACi JNJ-26481585. Eur J Hum Gen 2013;21:643-652.

107. Hamilton G, Gillingwater TH. Spinal muscular atrophy: going beyond the motor neuron. Trends Mol Med 2013;19:40-50.

108. Lee AJ, Awano T, Park GH, Monani UR. Limited phenotypic effects of selectively augmenting the SMN protein in the neurons of a mouse model of severe spinal muscular atrophy. PLoS One 2012;7:e46353.

109. Paez-Colasante X, Seaberg B, Martinez TL, Kong L, Sumner CJ, Rimer, M. Improvement of neuromuscular synaptic phenotypes without enhanced survival and motor function in severe spinal muscular atrophy mice selectively rescued in motor neurons. PLoS One 2013;8:e75866.

110. Iyer CC, McGovern VL, Murray JD, et al. Low levels of Survival Motor Neuron protein are sufficient for normal muscle function in the SMNDelta7 mouse model of SMA. Hum Mol Genet 2015;24: 6160-6173.

111. Bowerman M, Murray LM, Beauvais A, Pinheiro B, Kothary R. A critical smn threshold in mice dictates onset of an intermediate spinal muscular atrophy phenotype associated with a distinct neuromuscular junction pathology. Neuromuscul Disord 2012;22: 263-276. 